\title{
Plasma Flow Control
}

\author{
Ying-hong Li, Yun Wu, Hui-min Song, Hua Liang and Min Jia \\ Air Force Engineering University of China, \\ People's Repubilic of China
}

\section{Introduction}

Plasma flow control, based on the plasma aerodynamic actuation, is a novel active flow control technique to improve aircrafts' aerodynamic characteristics and propulsion efficiency. Plasma flow control has drawn considerable attention and been used in boundary layer acceleration, airfoil separation control, forebody separation control, turbine blade separation control, axial compressor stability extension, heat transfer and high speed jet control. Plasma aerodynamic actuator has many advantageous features including robustness, simplicity, low power consumption and ability for real-time control at high frequency.

In this chapter, the principle of plasma aerodynamic actuation and its application in subsonic and supersonic flow control was summarized. In order to better understand the underlying physical mechanism of plasma flow control and optimize the geometric configuration of the actuator, the characteristics of the plasma aerodynamic actuation, including gas temperature, electron density and temperature, induced body force, velocity and vorticity were investigated. Both wind tunnel experiments and computations were performed to investigate the flow control capability of plasma aerodynamic actuation for airfoil separation control, corner separation control, axial compressor stability extension and shock wave control.

\section{Plasma aerodynamic actuation}

The mechanism for plasma flow control can be summarized as momentum effect, shock effect, and chemical effect. Momentum effect induces near-surface flow velocity of 1-6 m/s. Shock effect induces local gas pressure or temperature rise near the electrode. Chemical effect adds new particles, such as ions, electrons, and excited particles, into the flow field. Surface dielectric barrier discharge plasma aerodynamic actuation is typically used in subsonic plasma flow control, while arc discharge plasma aerodynamic actuation is mostly used in supersonic and hypersonic plasma flow control. Plasma aerodynamic actuation generated by corona discharge, radiofrequency discharge, microwave discharge are also widely investigated.

\subsection{Surface dielectric barrier discharge plasma aerodynamic actuation}

A schematic of the dielectric barrier discharge plasma aerodynamic actuator is shown in Fig. 1. Most of the previous experimental and simulation studies mainly focused on the asymmetric dielectric barrier discharge plasma aerodynamic actuation excited by sinusoidal or sawtooth voltage waveforms at amplitude of $2-20 \mathrm{kV}$ and frequency of $1-100 \mathrm{kHz}$, which can be named as the microsecond discharge plasma aerodynamic actuation. In recent years, 
the plasma aerodynamic actuation generated by nanosecond pulsed dielectric barrier discharge has become a hot topic.

Synthetic measurements and analysis of the characteristics of the asymmetric surface dielectric barrier discharge plasma aerodynamic actuation were performed. Fig. 1 shows the schematic diagram of the asymmetric surface dielectric barrier discharge plasma aerodynamic actuator. The dielectric layer used is a RO4350B (Rogers Corporation) plate with a relative permittivity constant of 3.48. The electrodes are made of copper, covered with a thin layer of lead-tin film. The plasma aerodynamic actuator is driven by a high frequency high voltage power supply (CTP-2000K, Suman Electronics). The output voltage range and frequency range of the power supply are $0 \sim 40 \mathrm{kV}$ and $6 \sim 40 \mathrm{kHz}$ respectively.

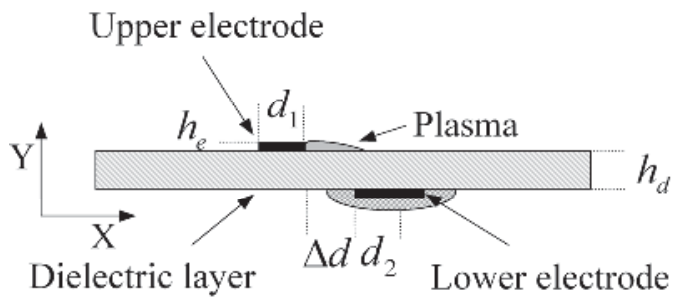

Fig. 1. A schematic of the asymmetric surface dielectric barrier discharge plasma aerodynamic actuator

The experimental arrangement is shown in Fig. 2. Synthetic measurements are applied to measure both the plasma and the induced flow characteristics of the plasma aerodynamic actuation. The applied voltage and the total discharge current are measured by a high voltage probe (P6015A, Tektronix Inc.) and a current probe (TCP312+TCPA300, Tektronix Inc.). Signals are recorded on an oscilloscope (DPO4104, Tektronix Inc.). Optical emission spectroscopy is measured by a $0.5 \mathrm{~m}$ monochrometer (TRIAX550, Jobin Yvon Inc.) through an optical fiber collector, $1 \mathrm{~cm}$ over the surface of the dielectric layer. The detector of the monochrometer is a set of photon counting system (Model 76915, Oriel Inc.). The slit width and the calibrated resolution in this case are $20 \mu \mathrm{m}$ and $0.05 \mathrm{~nm}$. The emission intensity is averaged temporally and spatially. Body force induced by the plasma aerodynamic actuation is measured by a electronic balance(ABS 204-S, Mettler Toledo Inc.). Velocity and vorticity induced by the plasma aerodynamic actuation is measured by Particle Image Velocimetry (Lavision). The air is seeded by vaporization of mineral oil with a mean size of about $0.3 \mu \mathrm{m}$.

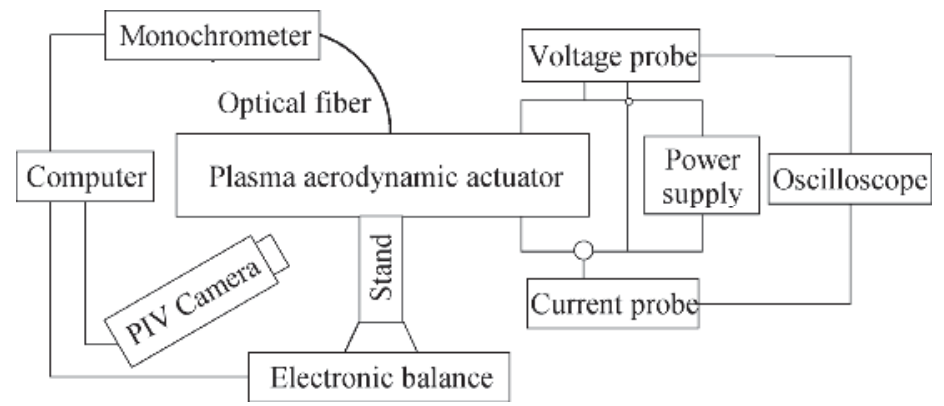

Fig. 2. Schematic diagram of the experimental arrangement 


\subsubsection{Characteristics of the plasma}

Optical emission spectroscopy(OES), as a simple and non-intrusive diagnostic method, is playing a very important role in providing qualitative and quantitative information of plasma properties atmospheric pressure air plasmas. Several plasma parameters, including gas temperature, vibrational temperature, electron temperature and density, have been successfully characterized by OES method in this study. The major spectra come from the second positive system (SPS) of $\mathrm{N}_{2}\left(\mathrm{C}^{3} \Pi_{\mathrm{u}} \rightarrow \mathrm{B}^{3} \Pi_{\mathrm{g}}\right)$, and the first negative system (FNS) of $\mathrm{N}_{2}{ }^{+}\left(\mathrm{B}^{2} \Sigma_{\mathrm{u}}{ }^{+} \rightarrow X^{2} \Sigma_{\mathrm{g}}{ }^{+}\right)$. The relative concentration of $\mathrm{N}_{2}\left(\mathrm{C}^{3} \Pi_{\mathrm{u}}\right)$ is much greater than that of $\mathrm{N}_{2}{ }^{+}\left(\mathrm{B}^{2} \Sigma_{\mathrm{u}}{ }^{+}\right)$. The emitting species of $\mathrm{N}_{2}\left(\mathrm{C}^{3} \Pi_{\mathrm{u}}\right)$ and $\mathrm{N}_{2}{ }^{+}\left(\mathrm{B}^{2} \Sigma_{\mathrm{u}}{ }^{+}\right)$mainly come from the following excitation processes from the ground state of $\mathrm{N}_{2}$.

$$
\begin{gathered}
e+N_{2}(X) \rightarrow e+N_{2}(C) \quad\left(E_{t h}=11 \mathrm{eV}\right) \\
e+N_{2}(X) \rightarrow e+e+N_{2}^{+}(B) \quad\left(E_{t h}=19 \mathrm{eV}\right)
\end{gathered}
$$

Other main dynamics/kinetics processes in this surface discharge include[24-28]:

$$
\begin{gathered}
e+N_{2}(X, v) \rightarrow e+N_{2}(X, \omega) \\
N_{2}(X, v)+N_{2}(X, \omega) \rightarrow N_{2}(X, v-1)+N_{2}(X, \omega+1) \\
N_{2}(X, v)+N_{2} \rightarrow N_{2}(X, v-1)+N_{2} \\
N_{2}(C)+N_{2}, O_{2} \rightarrow N_{2}, O_{2}+\text { product } \\
N_{2}(C) \rightarrow N_{2}(B)+h v \\
N_{2}^{+}(B)+N_{2}, O_{2} \rightarrow N_{2}, O_{2}+\text { product } \\
N_{2}^{+}(B) \rightarrow N_{2}^{+}(X)+h v
\end{gathered}
$$

In atmospheric discharge condition, the gas temperature can be estimated by the rotational temperature of a molecular because the rotational energy levels are closely spaced $\left(10^{-3} \mathrm{eV}\right)$ and allow for rapid energy transfer between the two energy modes. The population distribution in the rotational energy level of $\mathrm{N}_{2}$ is also believed to fit Boltzmann distribution due to the dense collisions between molecules. Here, we obtained the rotational temperature of $\mathrm{N}_{2}$ through fitting the $N_{2}$ second positive system band from $378 \mathrm{~nm}$ to $381 \mathrm{~nm}$ for the $380.5 \mathrm{~nm}\left(\mathrm{v}^{\prime}=0, \mathrm{v}^{\prime \prime}=2\right) \mathrm{N}_{2}$ line. By assuming a rotational temperature and considering the dipole radiation probability and the response function of the monochrometer, one can calculate the profile of a certain emission band. The actual rotational temperature $\left(T_{\mathrm{r}}\right)$ can be determined through comparing the experimental measurement and theoretical calculation. In this surface discharge, the vibrational excitation of nitrogen seems to be the most important. $\mathrm{N}_{2}\left(\mathrm{C}^{3} \Pi_{\mathrm{u}}\right)$, which is not a metastable state, is generated from the ground-state electron impact excitation and the cascading effect is not important for the $\mathrm{N}_{2}\left(\mathrm{C}^{3} \Pi_{\mathrm{u}}\right)$ state population. Therefore, the vibrational temperature can be determined according to the ratio of two lines in the $\mathrm{N}_{2}(\mathrm{C})$ second positive system. The spectra lines at $371.1 \mathrm{~nm}$ and $380.5 \mathrm{~nm}$ are selected to calculate the vibrational temperature $\left(T_{\mathrm{v}}\right)$, as follows: 


$$
\frac{I_{371.1 \mathrm{~nm}}}{I_{380.5 \mathrm{~nm}}}=1.1384 \cdot \exp \left(-0.4952 / T_{v}\right)
$$

When the applied voltage amplitude and driving frequency are $10 \mathrm{kV}$ (peak to peak) and $23 \mathrm{kHz}$ respectively, the rotational and vibrational temperatures of $\mathrm{N}_{2}\left(\mathrm{C}^{3} \Pi_{\mathrm{u}}\right)$ are $500 \mathrm{~K}$ and $0.22 \mathrm{eV}$ respectively. The rotational temperature is insensitive to the applied voltage and the driving frequency. The vibrational temperature also shows minor dependence on the applied voltage and the driving frequency, as shown in Fig. 3.

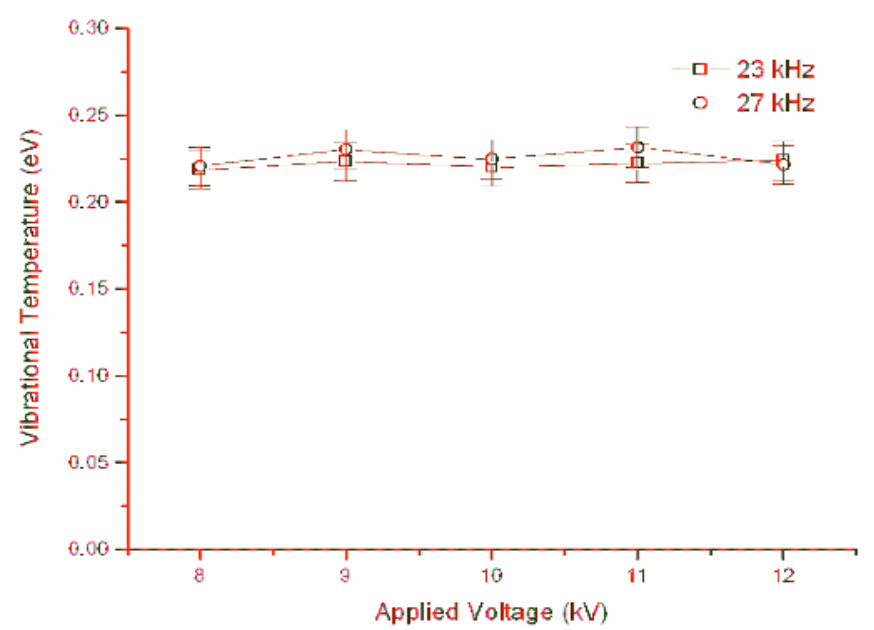

Fig. 3. Vibrational temperature versus the applied voltage

The $0 \mathrm{D}$ rate balance equation for the concentration, $n_{\mathrm{v}}$, of molecules in the vth vibrational level of the ground state is

$$
\begin{aligned}
\frac{d n_{v}}{d t}= & n_{e} \sum_{w \neq v} n_{w} C_{w}^{v}-n_{e} n_{v} \sum_{w \neq v} C_{v}^{w}+n_{v-1} \sum_{w} n_{w+1} Q_{v, v-1}^{w+1, w} \\
& +n_{v+1} \sum_{w} n_{w} Q_{v+1, v}^{w, w+1}-n_{v}\left(\sum_{w} n_{w+1} Q_{v, v+1}^{w+1, w}+\sum_{w} n_{w} Q_{v, v-1}^{w, w+1}\right) \\
& +n_{v+1} \sum_{w} n_{w} Q_{v+1}^{v}-n_{v} \sum_{w} n_{w} Q_{v}^{v-1}
\end{aligned}
$$

Here $Q$ and $C$ are rate coefficients of collision.

The rate balance equation for each excited state is, for state $\mathrm{N}_{2}(\mathrm{C})$,

$$
\frac{d n_{C}}{d t}=n_{e} n_{N_{2}} Q_{C}-A_{C} n_{C}-n_{C} n_{N_{2}} Q_{N_{2}}-n_{C} n_{O_{2}} Q_{O_{2}}
$$

for state $\mathrm{N}_{2}{ }^{+}(\mathrm{B})$,

$$
\frac{d n_{B+}}{d t}=n_{e} n_{N_{2}} Q_{B+}-A_{B+} n_{B+}-n_{B+} n_{N_{2}} Q_{N_{2}}-n_{B+} n_{O_{2}} Q_{O_{2}}
$$


Here $A$ is the Einstein coefficient.

The intensity ratio of optical emission line from FNS to SPS is strongly dependent on the electron temperature or the energy distribution function for the electrons whose energy is more than $11 \mathrm{eV}$, because the excited thresholds of the two processes have a difference of about $8 \mathrm{eV}$. According to equation (12) and (13), electron temperature( $\left.T_{\mathrm{e}}\right)$ can be calculated using the intensity ratio of $391.4 \mathrm{~nm}$ and $380.5 \mathrm{~nm}$ :

$$
\frac{I_{391.4 n m}}{I_{380.5 n m}}=K_{0} \cdot\left(T_{e}\right)^{C_{0}} \cdot \exp \left(-\frac{E_{0}}{T_{e}}\right)
$$

As shown in equation (12) and (13), the relative intensity of different vibrational levels of $\mathrm{N}_{2}(\mathrm{X})$ is mainly determined by the electron density. According to Frank-Condon principle, the vibrational energy level distribution of $\mathrm{N}_{2}(\mathrm{C})$ can be revealed using the changes in vibrational energy level distribution of $\mathrm{N}_{2}(X)$. Therefore, the ionization rate $(n)$ can be calculated using the intensity ratio of $371.1 \mathrm{~nm}$ and $380.5 \mathrm{~nm}$ :

$$
\frac{I_{371.1 n m}}{I_{380.5 n m}}=C_{0}+C_{1} \log _{10}(n)+C_{2}\left(\log _{10} n\right)^{2}
$$

The electron temperature and electron density are $1.63 \mathrm{eV}$ and $1.1 \times 10^{11} \mathrm{~cm}^{-3}$ respectively with the applied voltage of $10 \mathrm{kV}$. The driving frequency is $23 \mathrm{kHz}$. At one atmospheric pressure, electron temperature is usually $1 \sim 2 \mathrm{eV}$ because of the frequent collisions between electrons and molecules. The variations of average electron temperature and density with the applied voltage are shown in Fig. 4. It can be concluded that the average electron density and temperature have minor dependence on the applied voltage and its frequency. The electron temperature of a dielectric barrier discharge is strongly affected by the gas pressure because the collisionless free path of the electrons mainly determines the energy obtained by the electron from the electric field. The frequent collisions between electrons and molecules govern the discharge process at one atmosphere.

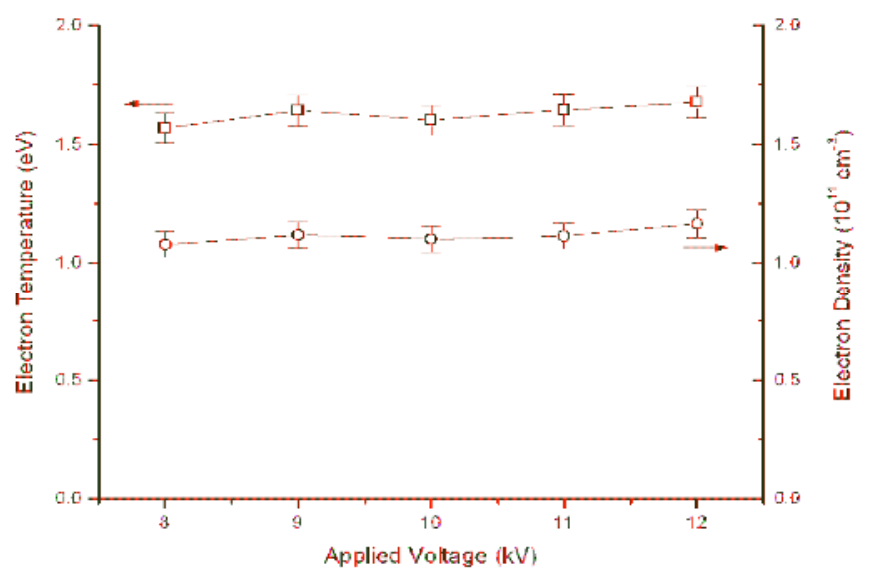

Fig. 4. Electron temperature and density versus the applied voltage 


\subsubsection{Characteristics of the induced flow}

The induced body force in $X$ direction versus the applied voltage is shown in Fig. 5. The driving frequency is $23 \mathrm{kHz}$. When the applied voltage increases from $8 \mathrm{kV}$ to $12 \mathrm{kV}$, the body force increases from $11 \mathrm{mN}$ to $65 \mathrm{mN}$.

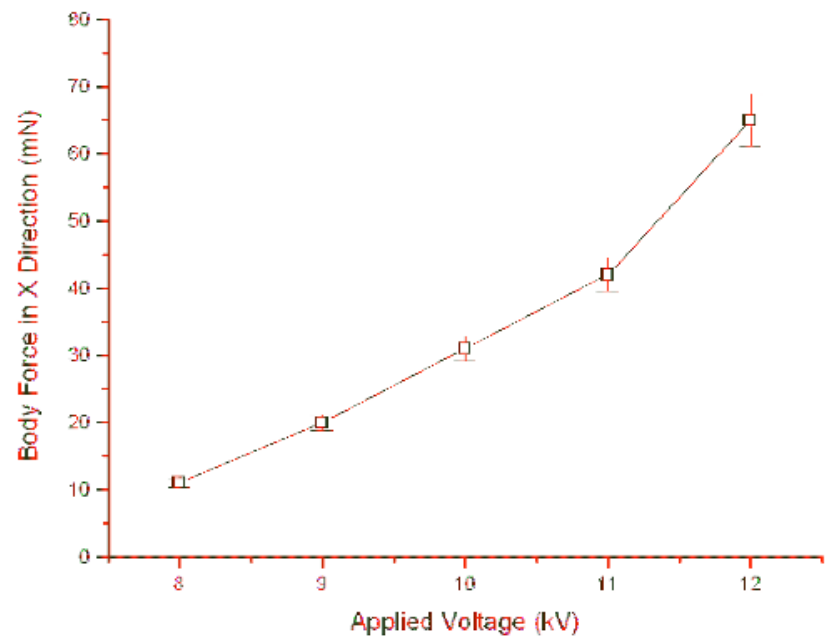

Fig. 5. Body force induced by the plasma aerodynamic actuation

Starting vortex and directed wall jet induced by the steady plasma aerodynamic actuation in static air are shown in Fig. 6 . The starting vortex exists for about $1 \mathrm{~s}$ and then directed wall jet is formed, about $70 \mathrm{~mm}$ downstream of the upper electrode. The induced flow velocity is $3 \mathrm{~m} / \mathrm{s}$ when the applied voltage and the driving frequency are $10 \mathrm{kV}$ and $23 \mathrm{kHz}$ respectively.

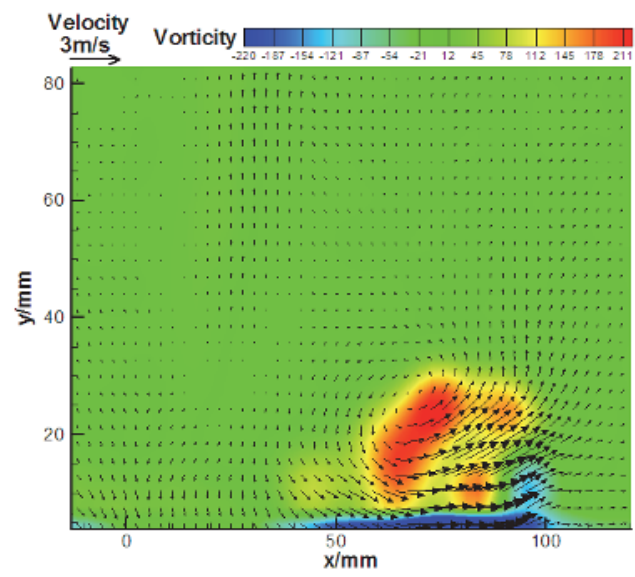

(a) $0.3 \mathrm{~s}$

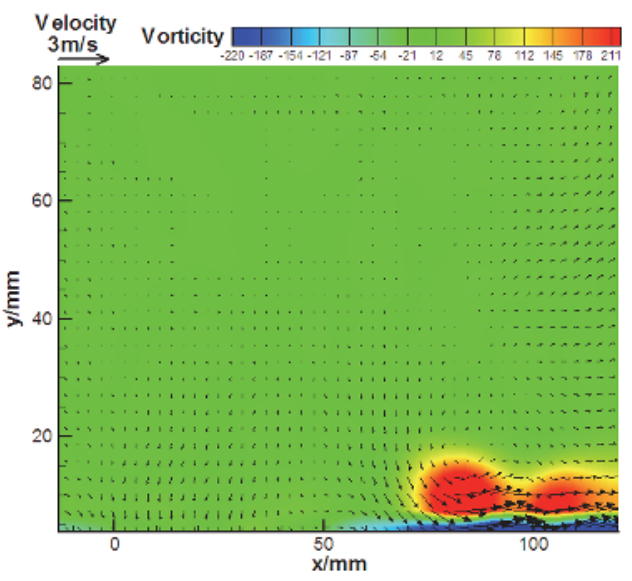

(b) $2.5 \mathrm{~s}$

Fig. 6. Velocity and vorticity of starting vortex induced by steady plasma aerodynamic actuation 
When the unsteady plasma aerodynamic actuation is on, the starting vortex is stronger and exists for a longer time, which is about $4 \mathrm{~m} / \mathrm{s}$. Then the directed wall jet along with many small vortexes is also formed, about $50 \mathrm{~mm}$ downstream of the upper electrode, as shown in Fig. 7. The duty cycle and excitation frequency are $70 \%$ and $190 \mathrm{~Hz}$ respectively. The unsteady plasma aerodynamic actuation induces much more vortex into the flow field.

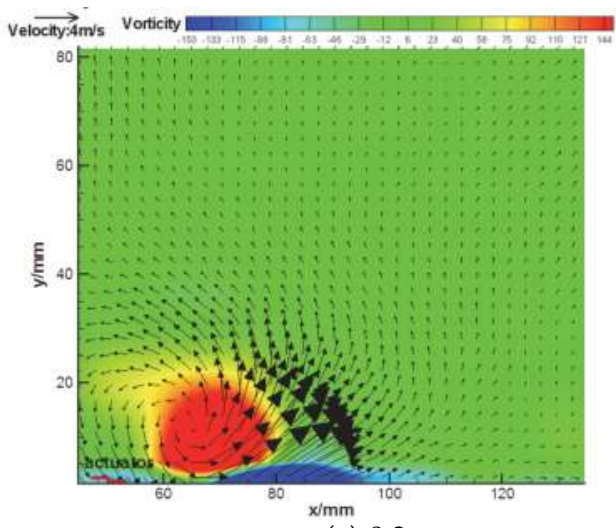

(a) $0.3 \mathrm{~s}$

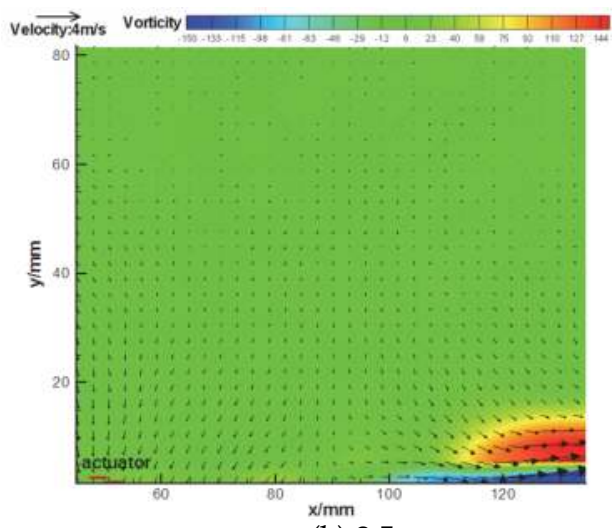

(b) $2.5 \mathrm{~s}$

Fig. 7. Velocity and vorticity of starting vortex induced by unsteady plasma aerodynamic actuation

When the voltage waveform is a nanosecond pulse, not the sinusoidal pulse, the induced flow direction changes remarkably. The induced flow direction by nanosecond discharge plasma aerodynamic actuation is not parallel, but vertical to the dielectric layer surface(see Fig. 8). The voltage pulse has a full wave at half maximum (FWHM) of $190 \mathrm{~ns}$ and a rise time of $450 \mathrm{~ns}$. The peak voltage and frequency are $10 \mathrm{kV}$ and $1 \mathrm{kHz}$, respectively.

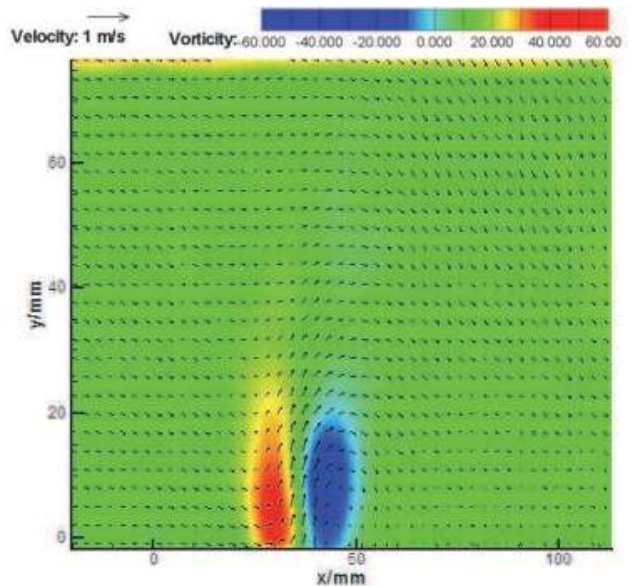

(a) $0.3 \mathrm{~s}$

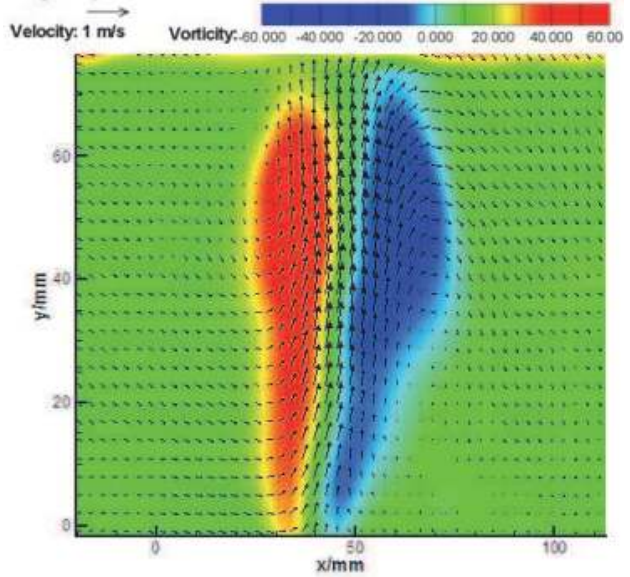

(b) $2.5 \mathrm{~s}$

Fig. 8. Velocity and vorticity of starting vortex induced by nanosecond discharge plasma aerodynamic actuation 


\subsection{Arc discharge plasma aerodynamic actuation}

A schematic of the arc discharge plasma aerodynamic actuator is shown in Fig. 9. A dc surface arc discharge is generated between the anode and the cathode. In order to generate a nonuniform electric field that can reduce gas breakdown voltage, a cylindrical structure is designed for the electrodes. The electrodes are oriented in the spanwise direction, which means that the flow direction is perpendicular to the discharge current direction.

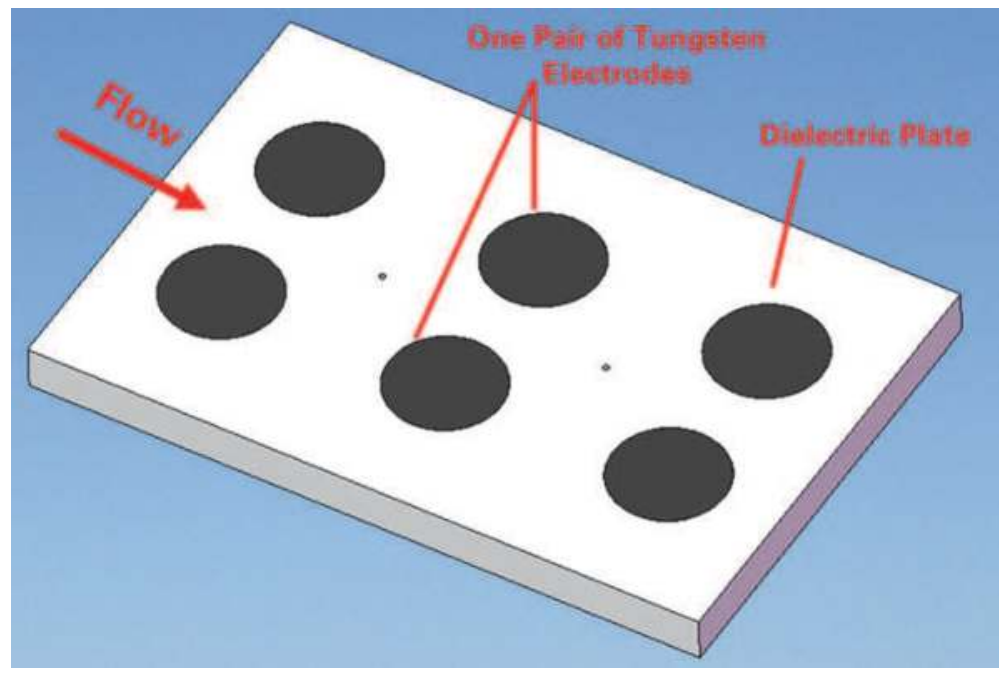

Fig. 9. A schematic of the arc discharge plasma aerodynamic actuator

The power supply consists of a high-voltage pulse circuit and a high-voltage dc circuit. The output voltage of the pulse circuit can reach $90 \mathrm{kV}$, which is used for electrical breakdown of the gas. The dc circuit is the $3 \mathrm{kV}-4 \mathrm{~kW}$ power source, which is used to ignite the arc discharge. The plasma aerodynamic actuator consists of graphite electrodes and boron-nitride (BN) ceramic dielectric material. Three pairs of graphite electrodes are designed with the cathode-anode interval of $5 \mathrm{~mm}$ and the individual electrode is designed as a cylindrical structure which is embedded in the BN ceramic. The arc discharge voltage and current are monitored by a voltage probe (P6015A, Tektronix Inc.) and a current probe with a signal amplifier (TCP312+TCPA300, Tektronix Inc.), respectively. The two signals are measured by a four-channel digital oscilloscope (DPO4104, Tektronix Inc.).

The voltage, current and power measurements are shown in Fig. 10. The gas breakdown voltage between the graphite electrodes is about $2 \mathrm{kV}$ and when the input voltage provided by the power supply exceeds this value, arc discharge happens. At the instant of gas breakdown, voltage decreases from $2 \mathrm{kV}$ to about $300 \mathrm{~V}$ and current increases to about $1 \mathrm{~A}$. The discharge power is calculated as $300 \mathrm{~W}$. Then the voltage holds at $300 \mathrm{~V}$, but the current decreases gradually. After about $0.5 \mathrm{~s}$, the current sustains at about $440 \mathrm{~mA}$ and the discharge power holds at about $130 \mathrm{~W}$. The arc discharge can be separated into two phases, which correspond to the strong pulsed breakdown process and the steady discharge process, respectively. 

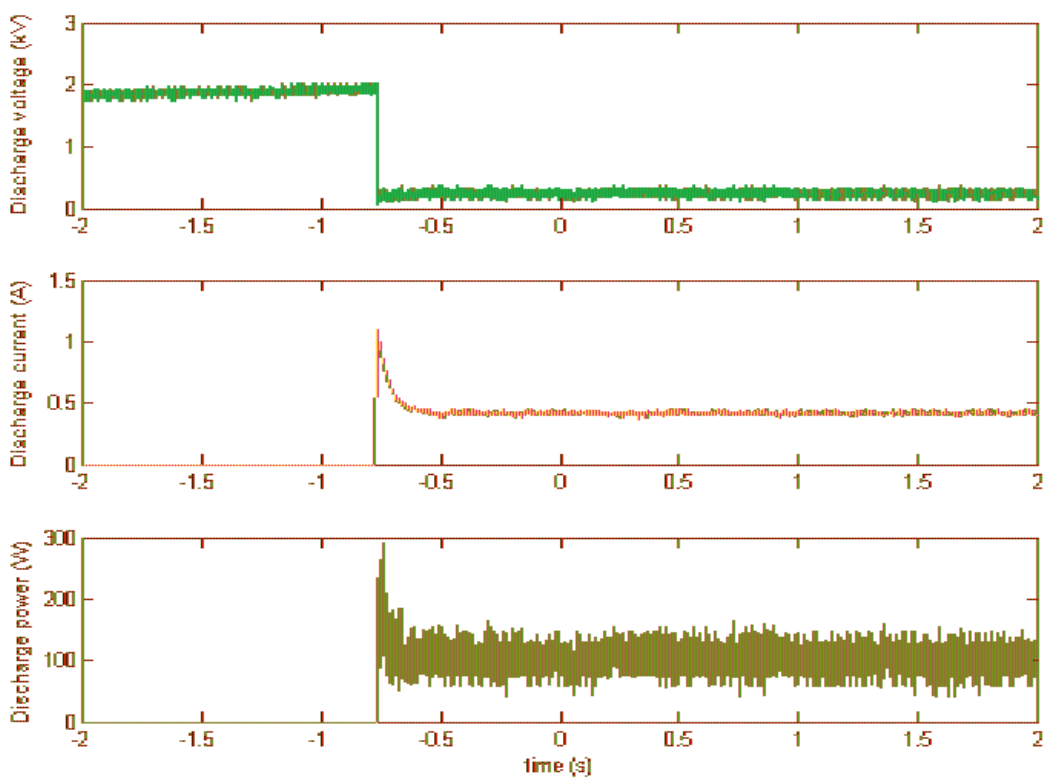

Fig. 10. Electrical characteristics of arc discharge

\section{Subsonic plasma flow control}

Surface dielectric barrier discharge was proved effective in subsonic plasma flow control. A great number of papers devoted to subsonic plasma flow control have appeared in the past ten years. The use of dielectric barrier discharge for flow control has been demonstrated in many applications. Examples include boundary layer acceleration, transition delay, lift augmentation on wings, separation control for low-pressure turbine blades, jet mixing enhancement, plasma flaps and slats, leading-edge separation control on wing sections, phased plasma arrays for unsteady flow control, and control of the dynamic stall vortex on oscillating airfoils.

\subsection{Airfoil flow separation control}

More than $70 \%$ lift force of aircraft is produced by wings. The lift-to-drag ratio and stall characteristic of the wing is of vital importance to the takeoff distance and climbing speed and the flight quality of the aircrafts. In order to enhance the manoeuvrability and flexibility of the aircrafts, large angle of attack is used frequently. New technology should be employed into the development of aircrafts of the next generation. Active flow control technologies are considered to be the most promising technology in the $21^{\text {th }}$ century.

\subsubsection{Flow separation control using microsecond and nanosecond discharge}

Flow separation control by microsecond and nanosecond discharge plasma aerodynamic actuation was presented. The control effects influenced by various actuation parameters were investigated. 
The airfoil used was a NACA 0015. This shape was chosen because it exhibits well-known and documented steady characteristics as well as leading-edge separation at large angles of attack. The airfoil had a $12 \mathrm{~cm}$ chord and a $20 \mathrm{~cm}$ span. The airfoil was made of Plexiglas. Twelve pressure ports were used to obtain the pressure distribution along the model surface. Fig. 11 shows location of the pressure ports on the model's surface. Three pairs of plasma aerodynamic actuators were mounted on the suction side of the airfoil. The actuators were positioned $2 \%$ and $20 \%$ and $45 \%$ cord length of the airfoil. The plasma aerodynamic actuators were made from two $0.018 \mathrm{~mm}$ thick copper electrodes separated by $1 \mathrm{~mm}$ thick Kapton film layer. The electrodes were $4 \mathrm{~mm}$ in width and $120 \mathrm{~mm}$ in length. They were arranged just in the asymmetric arrangement. A $1 \mathrm{~mm}$ recess was molded into the model to secure the actuator flush to the surface. The pressure distribution along the airfoil surface was obtained by a Scanivalve with 96 channels having a range of $\pm 11 \mathrm{kPa}$. A pitot static probe was mounted on the traversing mechanism. This was located at different positions downstream of the airfoil, on its spanwise centerline. Discrete points were sampled across the wake to determine the mean-velocity profile. The uncertainty of the measurement was calculated to be less than $1.5 \%$.

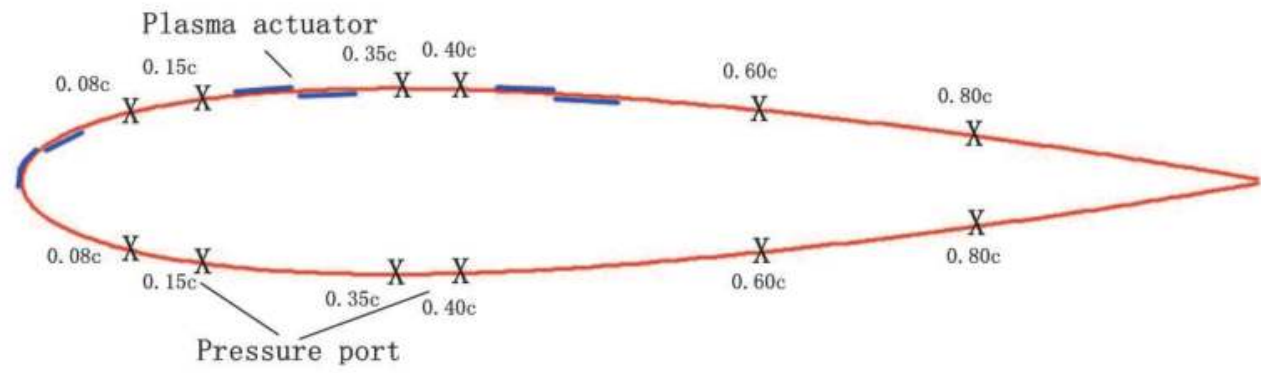

Fig. 11. A schematic of NACA 0015 airfoil with dielectric barrier discharge plasma aerodynamic actuator

The power supply used for microsecond discharge is $0-40 \mathrm{kV}$ and $6-40 \mathrm{kHz}$, respectively. The output voltage and the frequency range of the power supply used for nanosecond discharge are $5-80 \mathrm{kV}$ and $0.1-2 \mathrm{kHz}$, respectively. The rise time and full width half maximum (FWHM) are 190ns and 450ns, respectively.

The plasma aerodynamic actuation strength, which is related to the discharge voltage, is an important parameter in plasma flow control experiments. The flow control effects influenced by discharge voltage were investigated. Flow separates at the leading edge of the airfoil without discharge. The pressure distribution has a plateau from leading edge to trailing edge which corresponds to global separation from the leading edge. When the microsecond discharge voltage is $13 \mathrm{kV}$ and $14 \mathrm{kV}$, the flow separation can not be suppressed. As the microsecond discharge voltage increases to $15 \mathrm{kV}$, the actuation intensity increases and the flow separation is suppressed. There is a $34.0 \%$ lift force increase and a $25.3 \%$ drag force decrease when the discharge voltage is $15 \mathrm{kV}$. When the millisecond discharge voltage increases to $16 \mathrm{kV}$, there is a $35.1 \%$ lift force increase and a $25.5 \%$ drag force decrease. The control effects for discharge voltage of $15 \mathrm{kV}$ and $16 \mathrm{kV}$ are approximately the same. Thus, a threshold voltage exists for plasma aerodynamic actuation of different time scale. The flow separation can't be suppressed if the discharge voltage is 
less than the threshold voltage. When the flow separation is suppressed, the lift and drag almost unchanged when the discharge voltage increases. The initial actuation strength is of vital importance in plasma flow control. Once the flow separation is suppressed with a initial discharge voltage higher than the threshold voltage, the flow reattachment can be sustained even the discharge voltage was reduced to a value less than the threshold voltage, that is to say, the voltage to sustain the flow reattachment is lower than the voltage to suppress the flow separation in the same conditions. We can make use of the results by managing the discharge voltage properly. A higher discharge voltage can be used to suppress the separation in the beginning, and then we can use a much lower discharge voltage to sustain the flow reattachment later. Not only the power consumption can be reduced obviously, but also the life-span and the reliability of the actuator can be increased greatly.

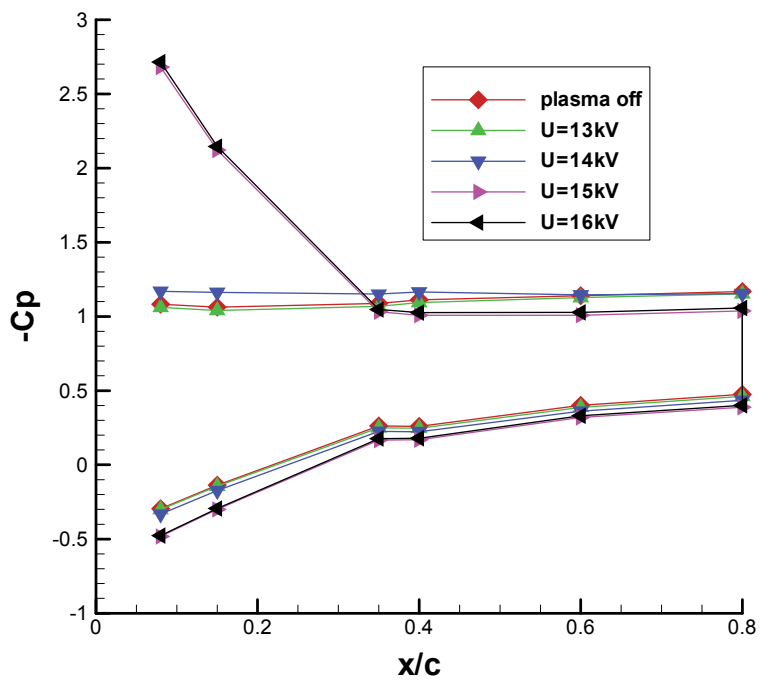

Fig. 12. Pressure distribution for microsecond discharge of different voltage $\left(\mathrm{a}=20^{\circ}, V_{\infty}=72 \mathrm{~m} / \mathrm{s}, \operatorname{Re}=5.8 \times 10^{5}\right)$

The frequency of nanosecond discharge is believed to be optimum when the Strouhal number $S_{t r}=f c_{\text {sep }} / v_{\infty}$ is near unity. The separation region length and inflow velocity are $100 \%$ chord length and $100 \mathrm{~m} / \mathrm{s}$ respectively. The Strouhal number is 1 when the pulse frequency is $830 \mathrm{~Hz}$. Experiments of different pulse frequency were made to determine if such an optimum frequency exists for the unsteady actuation used in controlling the airfoil flow separation.

The experimental results are shown in Fig. 13. It is found that there's an optimum pulse frequency in controlling the airfoil flow separation. The inflow velocity and the angle of attack are $100 \mathrm{~m} / \mathrm{s}$ and $25^{\circ}$ respectively. The duty cycle is fixed at $50 \%$. All three electrodes are switched on. The threshold voltage for different discharge frequency was shown Fig. 14 . When the pulse frequency is $830 \mathrm{~Hz}$, the threshold voltage to suppress the flow separation is only $10 \mathrm{kV}$ which is the lowest. When the pulse frequency is $200 \mathrm{~Hz}$ and $1500 \mathrm{~Hz}$, the threshold voltage is $13 \mathrm{kV}$ and $12 \mathrm{kV}$ respectively. 


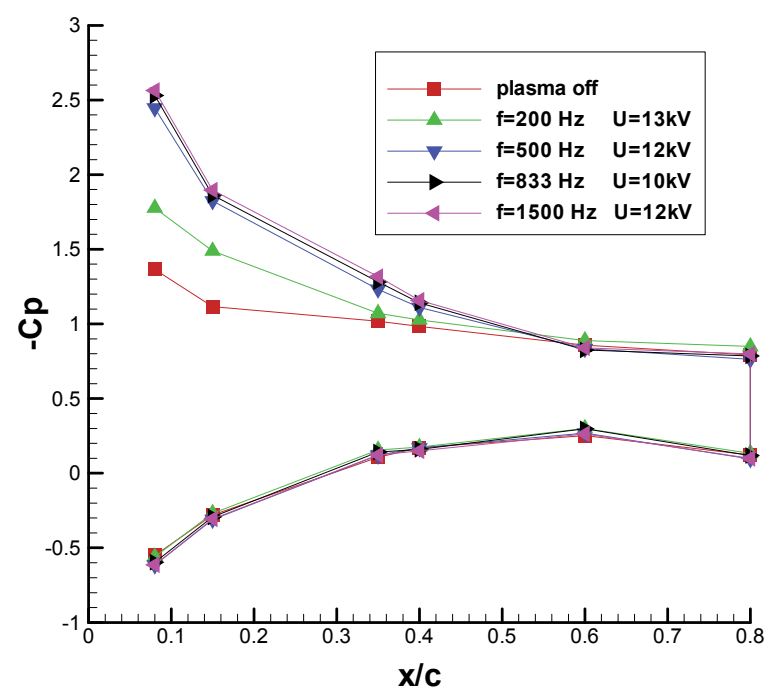

Fig. 13. Pressure distribution for nanosecond discharge of different frequency $\left(\mathrm{a}=20^{\circ}, V_{\infty}=100 \mathrm{~m} / \mathrm{s}, \operatorname{Re}=8.1 \times 10^{5}\right)$

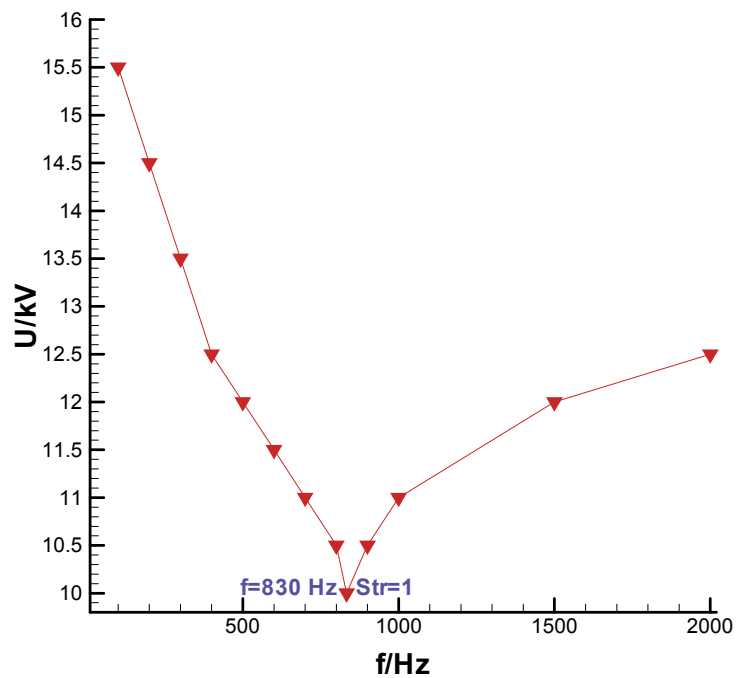

Fig. 14. The threshold voltage at different frequencies for nanosecond discharge $\left(V_{\infty}=100 \mathrm{~m} / \mathrm{s}, a=22^{\circ}, R e=8.1 \times 10^{5}\right)$

Plasma aerodynamic actuation of different time scales was used for flow separation control. The flow control ability for microsecond discharge and nanosecond discharge were analyzed. The pressure distribution along airfoil surface obtained in experiments for inflow velocity of $150 \mathrm{~m} / \mathrm{s}\left(\operatorname{Re}=12.2 \times 10^{5}\right)$ are presented in Fig. 15. The angle of attack is $25^{\circ}$, which is approximately $5^{\circ}$ past the critical angle of attack at the inflow velocity of $150 \mathrm{~m} / \mathrm{s}$ 
$\left(\operatorname{Re}=12.2 \times 10^{5}\right)$. The discharge frequency is fixed at $1600 \mathrm{~Hz}$. The discharge voltage for microsecond and nanosecond discharge is $17 \mathrm{kV}$ and $12 \mathrm{kV}$ respectively. When the nanosecond discharge is on, the flow is fully attached at the leading edge. The lift force increases by $22.1 \%$ and the drag force decreases by $17.4 \%$ with the actuation on. But the microsecond discharge can not suppress the flow separation. The flow still separates at the leading edge with microsecond plasma aerodynamic actuation. It indicates that the flow control ability for nanosecond discharge is stronger than that of the microsecond discharge. The nanosecond discharge is much more effective in leading edge separation control than microsecond discharge.

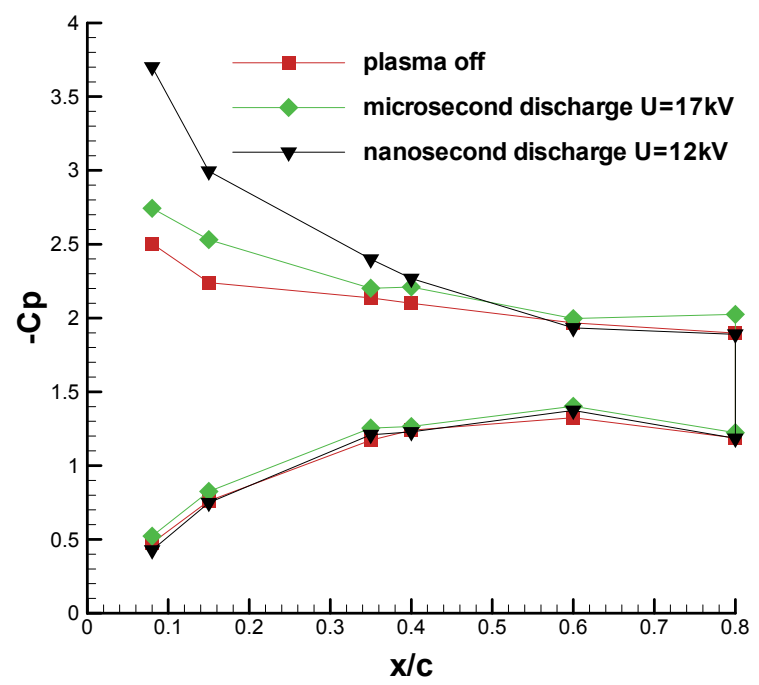

Fig. 15. Experimental results for microsecond and nanosecond discharge $\left(V_{\infty}=150 \mathrm{~m} / \mathrm{s}, a=25^{\circ}, R e=12.2 \times 10^{5}\right)$

\subsubsection{Flow separation control by spanwise nanosecond discharge}

The model used in this study was a NACA 0015 airfoil. Fig. 16 shows the geometry of the airfoil and the actuators. The actuator was made from two $0.018 \mathrm{~mm}$ thick copper electrodes separated by $1 \mathrm{~mm}$ thick Kapton film layer. The electrodes were $4 \mathrm{~mm}$ in width and $60 \mathrm{~mm}$ in length. They were arranged just in the asymmetric arrangement.

Experimental results for different angle of attacks (a) at the inflow velocity of $72 \mathrm{~m} / \mathrm{s}$ $\left(\operatorname{Re}=5.8 \times 10^{5}\right)$ are shown in Fig. 17. The discharge voltage and frequency of the nanosecond power supply were fixed at $13 \mathrm{kV}$ and $1000 \mathrm{~Hz}$ respectively. Experimental results show that spanwise nanosecond discharge aerodynamic actuation can suppress the flow separation effectively. The lift and drag coefficient are nearly unchanged with actuation when the angle of attack is less than $18^{\circ}$ or more than $24^{\circ}$. When the angle of attack is less than the critical value, there is nearly no flow separation on the airfoil surface. The effect of spanwise nanosecond discharge aerodynamic actuation can is not obvious. When the angle of attack is more than $24^{\circ}$, the flow separation on the airfoil surface is so aggressive that spanwise nanosecond discharge aerodynamic actuation can not suppress the flow separation on the suction side of the airfoil. So the lift and drag coefficients nearly the same. There is an 
obvious lift augmentation and drag reduction after actuation when the angle of attack is between $18^{\circ}$ and $24^{\circ}$. The lift coefficient is increased from 0.814 to 1.099 and the drag coefficient is decreased from 0.460 to 0.328 after actuation at the angle of attack $24^{\circ}$. The critical stall angle of attack for NACA 0015 airfoil increased from $18^{\circ}$ to $24^{\circ}$. When the angle of attack is $24^{\circ}$, there is a lift force augmentation of $30.2 \%$ and a drag force reduction of $22.1 \%$ after actuation.

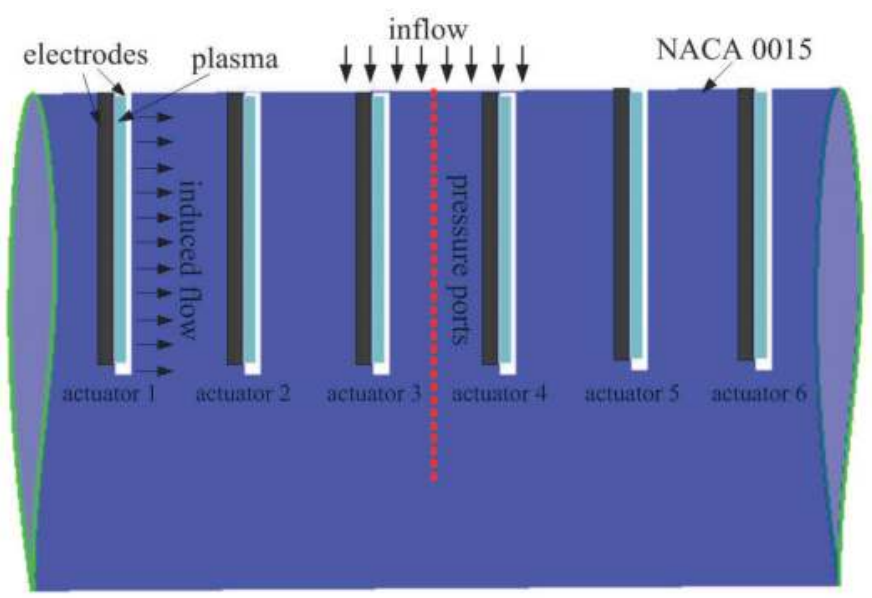

Fig. 16. Schematic drawing of the actuators on the airfoil

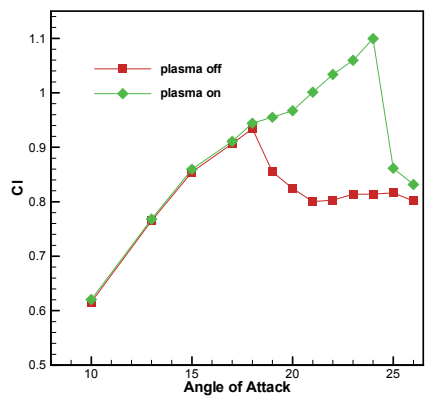

(a) Results of lift coefficient

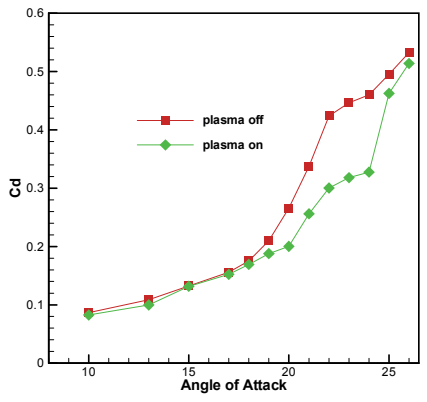

(b) Results of drag coefficient

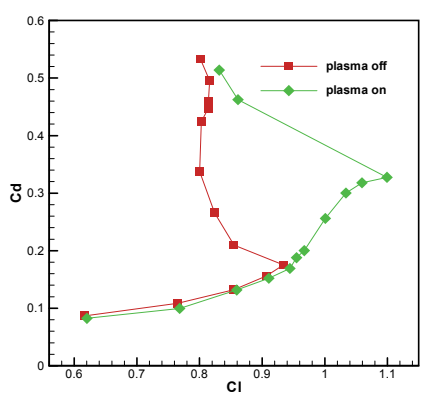

(c) Results of lift-to-drag ratio

Fig. 17. Experimental results at different angles of attack $\left(V_{\infty}=72 \mathrm{~m} / \mathrm{s}, R e=5.8 \times 10^{5}\right)$

The discharge frequency for microsecond discharge is in the orders of kilo hertz. Spanwise plasma aerodynamic actuation of different time scales was used for flow separation control. The flow control ability for microsecond discharge and nanosecond discharge were analyzed. The pressure distribution along airfoil surface obtained in experiments for inflow velocity of $66 \mathrm{~m} / \mathrm{s}\left(R e=5.3 \times 10^{5}\right)$ and $100 \mathrm{~m} / \mathrm{s}\left(R e=8.1 \times 10^{5}\right)$ are presented in Fig. 18 and Fig. 19. At the angle of attack $22^{\circ}$ and inflow velocity of $66 \mathrm{~m} / \mathrm{s}$ (Fig. 18), there is initial separated flow on the suction surface of the airfoil without discharge. The discharge voltage for microsecond and nanosecond discharge is $7 \mathrm{kV}$ and 
$12 \mathrm{kV}$ respectively. The discharge frequency is $1000 \mathrm{~Hz}$. The flow separation on the suction surface can be suppressed by both microsecond and nanosecond discharge actuation. The control effects are nearly the same for microsecond and nanosecond discharge. The spanwise plasma aerodynamic actuations result in a lift augmentation of $23.6 \%$ and a drag reduction of $25.6 \%$.

In Fig. 19, the angle of attack is $24^{\circ}$, which is approximately $4^{\circ}$ past the critical angle of attack at the inflow velocity of $100 \mathrm{~m} / \mathrm{s}\left(R e=5.8 \times 10^{5}\right)$. The discharge frequency is fixed at $1000 \mathrm{~Hz}$. The discharge voltage for microsecond and nanosecond discharge is $8.5 \mathrm{kV}$ and 12 $\mathrm{kV}$ respectively. When the nanosecond discharge is on, the flow is fully attached at the leading edge. The lift force increases by $25.3 \%$ and the drag force decreases by $20.1 \%$ with the actuation on. But the microsecond discharge can not suppress the flow separation. The flow still separates at the leading edge with microsecond plasma aerodynamic actuation. It indicates that the flow control ability for nanosecond discharge is stronger than that of the microsecond discharge. The nanosecond discharge actuation is much more effective in leading edge separation control than microsecond discharge actuation.

The dielectric layer will be destroyed when the discharge voltage is strong enough. Kapton is used as the dielectric in our experiments. The threshold voltage to destroy the Kapton layer is $8.5 \mathrm{kV}$ for microsecond discharge in our experiments. The actuators will be destroyed when the discharge voltage is more than $8.5 \mathrm{kV}$ for microsecond discharge. The threshold voltage to destroy the Kapton layer is $17 \mathrm{kV}$ for nanosecond discharge in our experiments.The instantaneous actuation intensity for nanosecond discharge is much stronger than microsecond discharge. So nanosecond discharge is more effective in flow control than microsecond discharge.

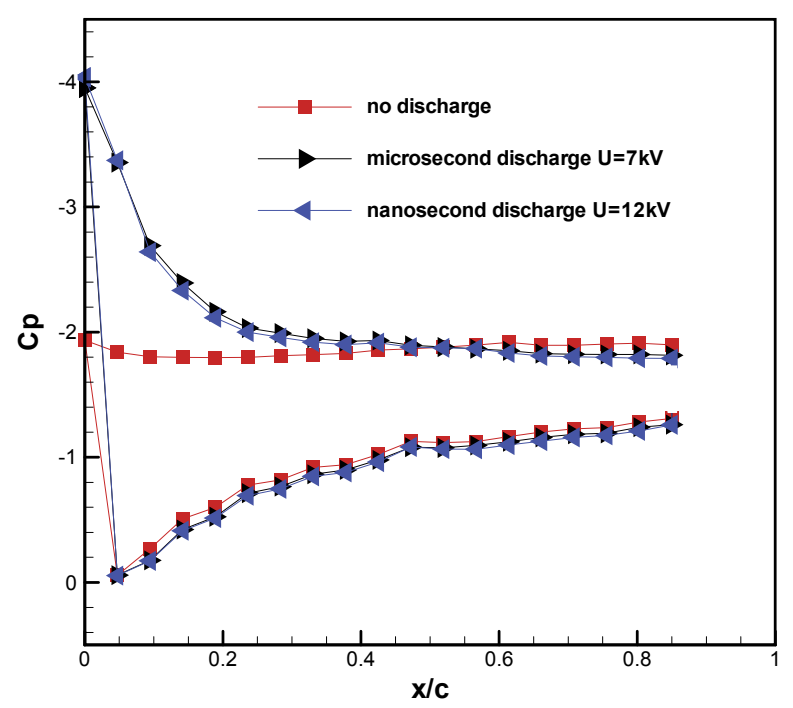

Fig. 18. Experimental results for microsecond and nanosecond discharge $\left(V_{\infty}=66 \mathrm{~m} / \mathrm{s}\right.$ and $\left.\mathrm{a}=22^{\circ} \operatorname{Re}=5.3 \times 10^{5}\right)$ 


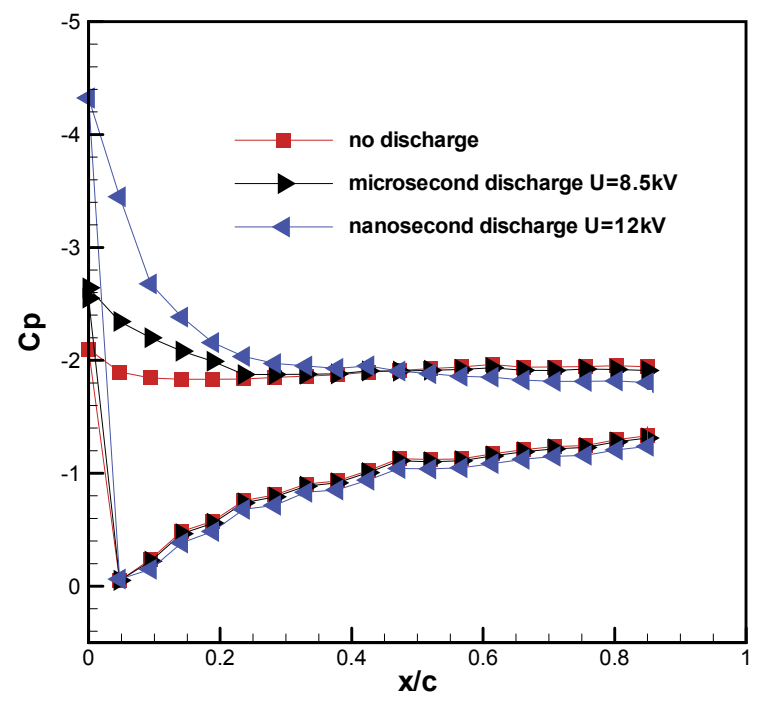

Fig. 19. Experimental results for microsecond and nanosecond discharge $\left(V_{\infty}=100 \mathrm{~m} / \mathrm{s}, a=24^{\circ}, R e=8.1 \times 10^{5}\right)$

\subsubsection{The mechanism of plasma shock flow control}

Based on our works, the principle of "plasma-shock-based flow control" was proposed. Energy should be released in extremely short time to intensify the instantaneous actuation strength, such as nanosecond discharge. Nanosecond discharge yields strong turbulence even shock waves which are act on the boundary layer. Shock wave produces stronger turbulent mixing of the flow, which can enhance momentum and energy exchange between the boundary layer and inflow greatly. High momentum fluid was brought into the boundary layer intermittently, enabling the flow to withstand the adverse pressure gradient without flow separation .The spirits of "plasma-shock-based flow control" lay in three aspects. Firstly, "Shock Actuation", nanosecond discharge should be used to increase the instantaneous discharge power. Nanosecond discharge induces strong local pressure or temperature rise in the boundary. Pressure or temperature rise result in strong pulse disturbance or shock waves in the boundary. Secondly, "Vortex control", shock wave disturbance induces vortex in the process of propagation. Vortex enhances energy and momentum mixing between boundary layer and inflow. The velocity of the boundary layer increase and the flow separation is suppressed. Thirdly, "Frequency Coupling", adjust the discharge frequency to the optimal response frequency in flow control. The optimal response frequency is the one which makes the Strouhal number equal to 1 . The plasma aerodynamic actuation work best at the optimal response frequency. Nanosecond discharge can increase the capability of plasma flow control effectively while its energy consumption can be reduced greatly.

For microsecond plasma aerodynamic actuation, the momentum effect may be the dominant mechanism. Microsecond plasma aerodynamic actuation induces near-surface boundary layer acceleration. Energy and momentum is added into the boundary layer, which enhances the ability to resist flow separation caused by adverse pressure gradient for boundary. But the maximum induced velocity for microsecond discharge is less than $10 \mathrm{~m} / \mathrm{s}$. 
The actuators will be destroyed if the discharge voltage is too high. The momentum added into the boundary layer by microsecond discharge is quite limited. The microsecond plasma aerodynamic actuation can only work effectively when the inflow velocity is several tens of meters per second.

The main mechanism for nanosecond discharge plasma flow control may be not momentum effect, since the induced velocity is less than $1 \mathrm{~m} / \mathrm{s}$. The velocity and vorticity measurements by the Particle Image Velocimetry show that, the flow direction is vertical, not parallel to the dielectric layer surface. The induce flow is likely to be formed by temperature and pressure gradient caused by nanosecond discharge other than energy exchange between charged and neutral particles. Thus, the main flow control mechanism for nanosecond plasma aerodynamic actuation is local fast heating due to high reduced electric field, which then induces shock wave and vortex near the electrode.

Experimental results indicate that nanosecond discharge is more effective in flow control than microsecond discharge. The latest study showed that nanosecond discharges have demonstrated an extremely high efficiency of operation for aerodynamic plasma actuators over a very wide velocity range $(\mathrm{Ma}=0.03-0.75)$. So shock effect is more important than momentum effect in plasma flow control.

\subsection{Corner separation control in a compressor cascade}

Control of the corner separation is one of the important ways of improving axial compressor stability and efficiency. Our approach to control the corner separation is based on the use of plasma aerodynamic actuation. Experiments were carried out on a low speed compressor cascade facility. Main cascade parameters are shown in Fig. 20. Only the middle blade was laid with the plasma aerodynamic actuator.

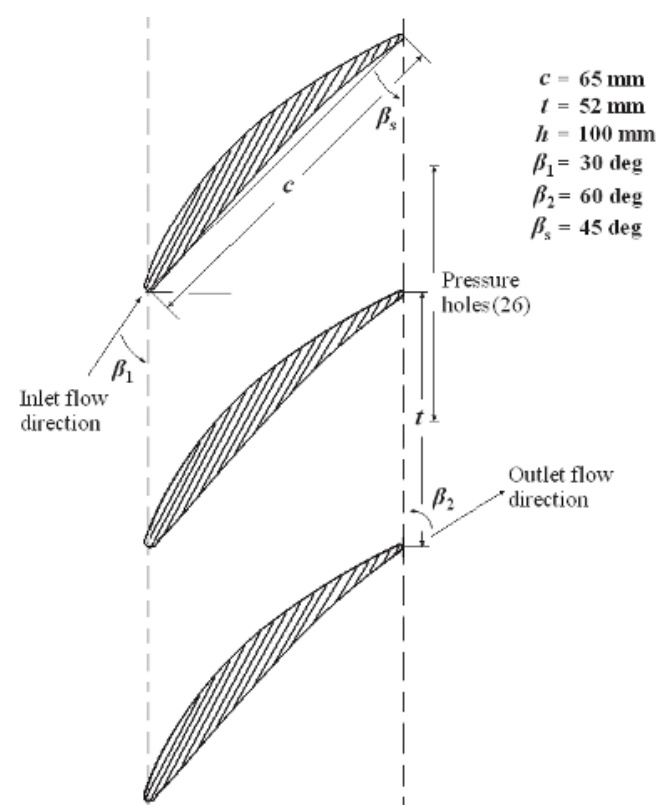

Fig. 20. Compressor cascade parameters 
Total pressure distributions at $10 \mathrm{~mm}$, which is $15 \%$ of the chord length, downstream of the blade trailing edge along the pitch direction at 50\%, 60\% and $70 \%$ blade spans were measured with and without the plasma aerodynamic actuation. A three-hole probe calibrated for pitch and yaw was used to measure the total pressure at the cascade exit. Two parameters, total pressure recovery coefficient $\sigma$ and the relative reduction of the total pressure loss coefficient $\delta(\omega)$, were used to quantify the performance improvement due to the plasma aerodynamic actuation.

The plasma aerodynamic actuator used in the present experiments consists of four electrode pairs, located at $5 \%, 25 \%, 50 \%$ and $75 \%$ of the chord length, respectively. The electrode pair at $5 \%$ of chord length is named as the 1 st electrode pair. A sketch of a blade with the actuator on the surface is shown in Fig. 21. The electrode thickness is not to scale in the figure.

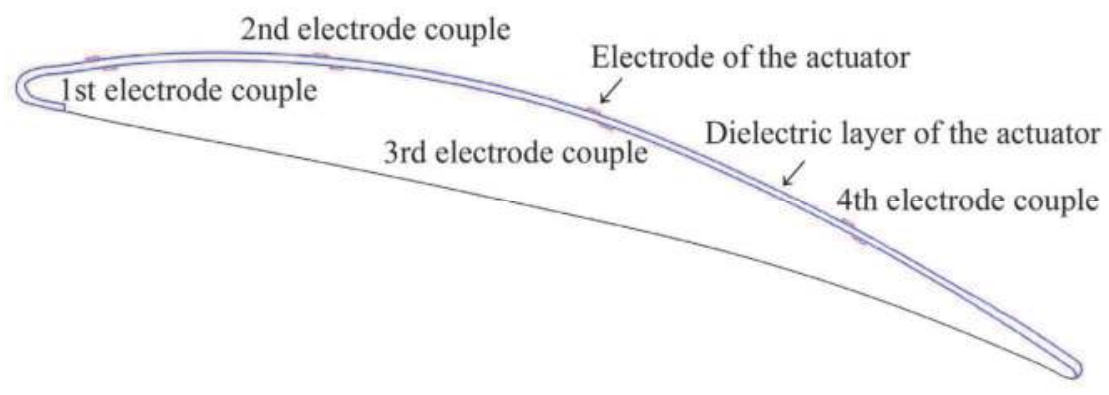

Fig. 21. A sketch of a blade with plasma aerodynamic actuator

The plasma aerodynamic actuator is driven by a high frequency high voltage power supply (CTP-2000M+, Suman Electronics). The output waveform is sine wave. The output ranges of the peak-to-peak voltage and the driving frequency of the power supply are $V_{\text {p-p }}=0 \sim 40 \mathrm{kV}$ and $F=6 \sim 40 \mathrm{kHz}$, respectively. The driving frequency is fixed at $23 \mathrm{kHz}$ in the experiments. The plasma aerodynamic actuator works at steady or unsteady mode in the experiments. In the steady mode, the actuator is operated at the ac frequency. In the unsteady mode of operation, the ac voltage is cycled off and on. Fig. 22 shows a typical signal sent to the plasma aerodynamic actuator during the unsteady actuation. Two important parameters of the unsteady plasma aerodynamic actuation are the excitation frequency $f$, and the duty cycle $a$, respectively.

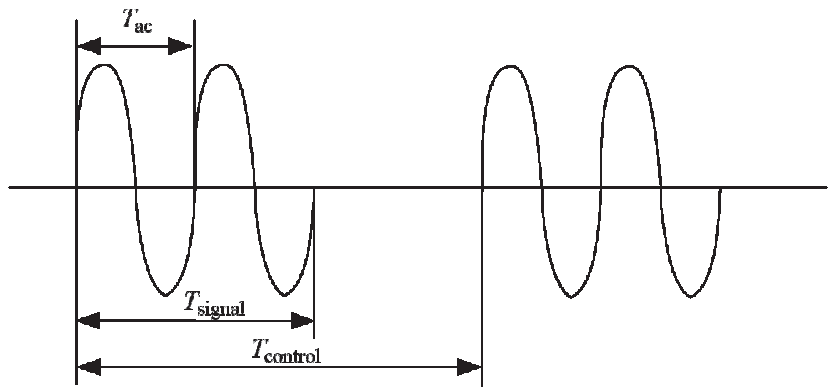

Fig. 22. The signal sent to the plasma aerodynamic actuator during unsteady excitation 


\subsubsection{Steady plasma flow control experiment results}

The mechanism of steady plasma aerodynamic actuation to control the corner separation may be that the actuation induces a time-averaged body force on the flow due to that the flow can't respond to such high frequency ( $23 \mathrm{kHz}$ in the experiments) disturbances. A wall jet, which is oriented in the mean flow direction, is produced to add momentum to the nearwall boundary layer near the flow separation location. The energized flow is able to withstand the adverse pressure gradient without separation. The directed wall jet governs the flow control effect of steady plasma aerodynamic actuation. When the electrode length is enlarged, the consumed power increases nonlinearly.

The location of the plasma aerodynamic actuation is a key parameter in plasma flow control experiments. Total pressure recovery coefficients with steady actuation at different locations are shown in Fig. 23.

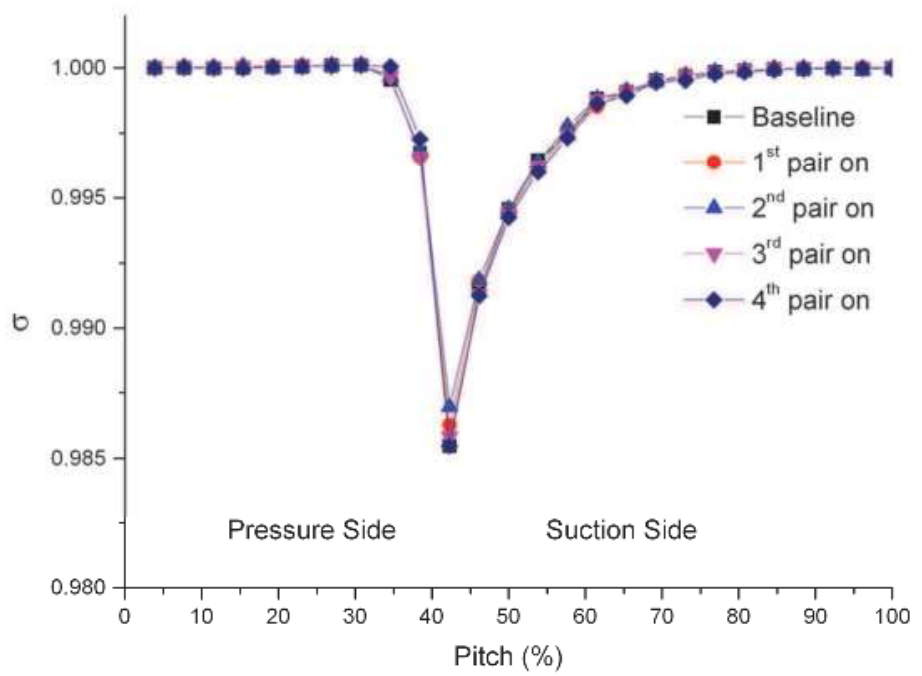

Fig. 23. Total pressure recovery coefficients with steady actuation at different locations $\left(\nu_{\infty}=50 \mathrm{~m} / \mathrm{s}, i=0 \mathrm{deg}, V_{\mathrm{p}-\mathrm{p}}=10 \mathrm{kV}, F=23 \mathrm{kHz}, 70 \% \mathrm{Span}\right)$

The applied peak-to-peak voltage and driving frequency are $V_{\mathrm{p}-\mathrm{p}}=10 \mathrm{kV}$ and $F=23 \mathrm{kHz}$, respectively. $\delta(\omega)_{\max }$ is $5.5 \%, 10.3 \%, 2.4 \%$ and $0.07 \%$ when the 1 st, $2^{\text {nd }}, 3^{\text {rd }}$ and $4^{\text {th }}$ electrode pair is switched on, respectively. The $2^{\text {nd }}$ electrode pair at $25 \%$ chord length is most effective and the control effect is as the same as that obtained by all four electrode pairs. The power dissipated by the $2^{\text {nd }}$ electrode pair is just $18.4 \mathrm{~W}$, about half of the power dissipated by all four electrode pairs. Therefore, the actuation location is vital to the control effect in corner separation control. In corner separation control by tailored boundary layer suction, the optimum slot should be long enough to be sure to remove the limiting streamline and the suction upstream of the corner separation location at the suction surface is most important for the control effect. Therefore, it can be inferred that the location of the $2^{\text {nd }}$ electrode pair is just upstream of the corner separation.

The plasma aerodynamic actuation strength is another important parameter in plasma flow control experiments. The body force increases with the voltage amplitude in proportion to 
the volume of plasma (ionized air) and the strength of the electric field gradient. As the applied peak to peak voltage increases from $8 \mathrm{kV}$ to $12 \mathrm{kV}, \delta(\omega)_{\max }$ increases from to $2.7 \%$ to $11.1 \%$, as shown in Fig. 24. The 2nd electrode pair at $25 \%$ chord length is switched on and the driving frequency is $23 \mathrm{kHz}$. The power dissipation increases from $8.4 \mathrm{~W}$ to $23.5 \mathrm{~W}$ when the applied peak to peak voltage increases from $8 \mathrm{kV}$ to $12 \mathrm{kV}$. When the applied voltage is less than $9 \mathrm{kV}$, the control effect is very tiny. When the applied voltage is higher than $10 \mathrm{kV}$, the control effect saturates and further increases in the voltage amplitude shows no evident benefit. Furthermore, higher voltage may lead to earlier destruction of the dielectric material, which is not desirable in the experiments.

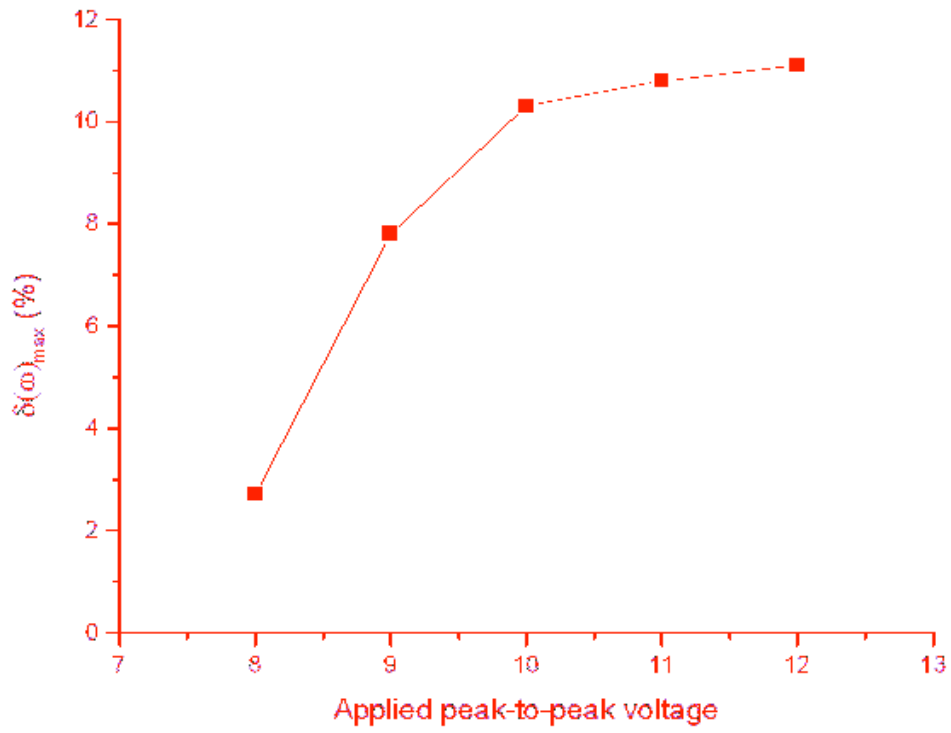

Fig. 24. Control effect with steady actuation of different applied voltages $\left(\nu_{\infty}=50 \mathrm{~m} / \mathrm{s}, i=0 \mathrm{deg}, F=23 \mathrm{kHz}, 70 \%\right.$ Span)

\subsubsection{Unsteady plasma flow control experiment results}

Optimization of the excitation mode based on coupling between the plasma aerodynamic actuation and the separated flow is one of the important ways of improving plasma flow control effect. It has been shown in the literature that the introduction of unsteady disturbances near the separation location can cause the generation of large coherent vortical structures that could prevent or delay the onset of flow separation. These structures are thought to intermittently bring high momentum fluid to the surface, enabling the flow to withstand the adverse pressure gradient without separation.

A sensitive study is performed to determine if such an optimum frequency exists for the unsteady actuation used in controlling the corner separation. Fig. 25 documents the relative reductions of maximum total pressure loss coefficient at $70 \%$ blade span for a range of excitation frequencies from $100 \mathrm{~Hz}$ to $1000 \mathrm{~Hz}$ when the duty cycle is fixed at $60 \%$. All four electrode pairs are switched on. The applied peak-to-peak voltage and driving frequency are $V_{\mathrm{p}-\mathrm{p}}=10 \mathrm{kV}$ and $F=23 \mathrm{kHz}$, respectively. 


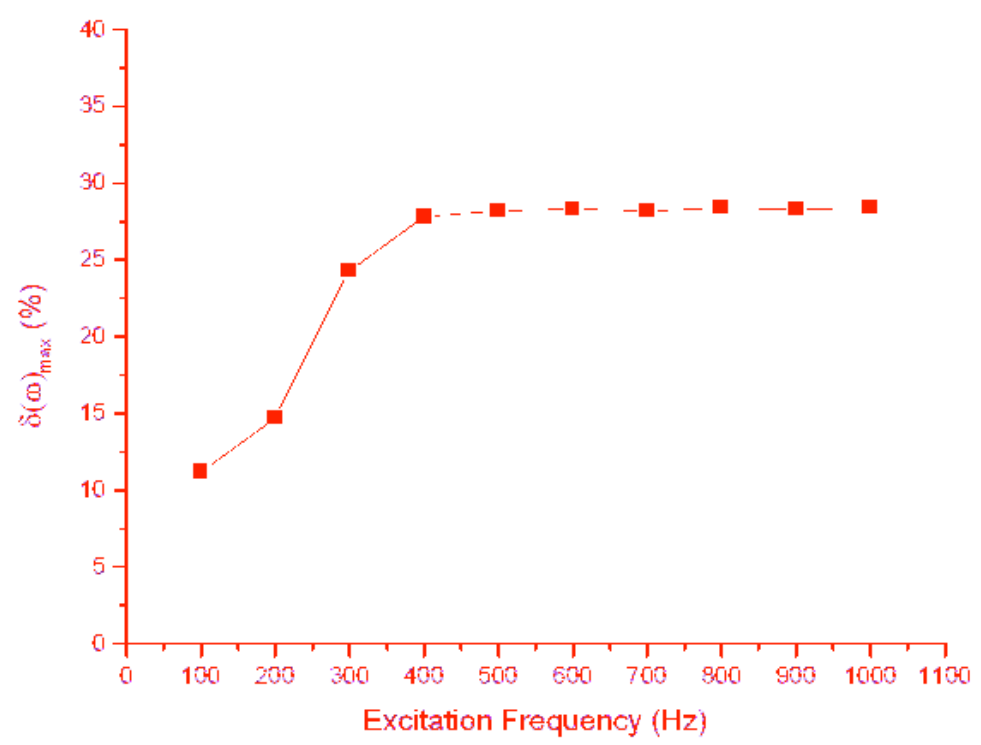

Fig. 25. Maximum relative reductions of total pressure loss coefficient with unsteady actuation of different duty cycles $\left(v_{\infty}=50 \mathrm{~m} / \mathrm{s}, i=0 \mathrm{deg}, V_{\mathrm{p}-\mathrm{p}}=10 \mathrm{kV}, F=23 \mathrm{kHz}, 70 \% \mathrm{Span}\right)$

When the excitation is $100 \mathrm{~Hz}, \delta(\omega)_{\max }$ is just $11.2 \%$, which is almost as same as the steady control effect that is $10.7 \%$. Along with the excitation frequency increasing, the control effect increases. When the excitation frequency is $400 \mathrm{~Hz}, \delta(\omega)_{\max }$ increases to $28 \%$. Thus, compared with the steady actuation, the unsteady actuation is much more effective and requires less power. When the excitation frequency is higher than $400 \mathrm{~Hz}$, the control effect saturates and further increases in the excitation frequency shows no evident benefit. The difference between steady and unsteady plasma aerodynamic actuation may be that, the unsteady pulsed operation allows the continuously generation of vortical structures, while the steady operation can't. Vortical structures in the flow field promote momentum transfer in the boundary layer in order to withstand separation. Under different duty cycles and excitation frequencies, the coupling between actuation and flow field leads to different flow control effects.

Each electrode pair is switched on to study the effect of the actuation location. The control effect of all four electrode pairs is almost as same as that obtained by the $2^{\text {nd }}$ electrode pair. The saturation frequency is also $400 \mathrm{~Hz}$. For the $2^{\text {nd }}$ electrode pair, the characteristic length is the remaining chord length downstream of the actuator, which is $75 \%$ chord length. Thus, the Strouhal number $S r=f \times C / \nu_{\infty}$ is 0.4 when the frequency and freestream velocity are $f=400 \mathrm{~Hz}$ and $v_{\infty}=50 \mathrm{~m} / \mathrm{s}$, respectively. When the Strouhal number exceeds 0.4, the control effect saturates in the unsteady plasma flow control experiments. In the separation control above a NACA 0015 airfoil with unsteady plasma aerodynamic actuation (Benard et al. 2009), the most effective actuation was performed with a Strouhal number of $S r$ ranging from 0.2 to 1.The optimum excitation frequency depends much on the flow separation state. Under different flow conditions, the optimum excitation frequency is also different. 
Fig. 26 documents the maximum relative reductions of total pressure loss coefficient for a range of unsteady duty cycles from $5 \%$ to $100 \%$ when the excitation frequency is fixed at 400 $\mathrm{Hz}$. All four electrode pairs are switched on. The applied peak-to-peak voltage and driving frequency are $V_{\mathrm{p}-\mathrm{p}}=10 \mathrm{kV}$ and $F=23 \mathrm{kHz}$, respectively.

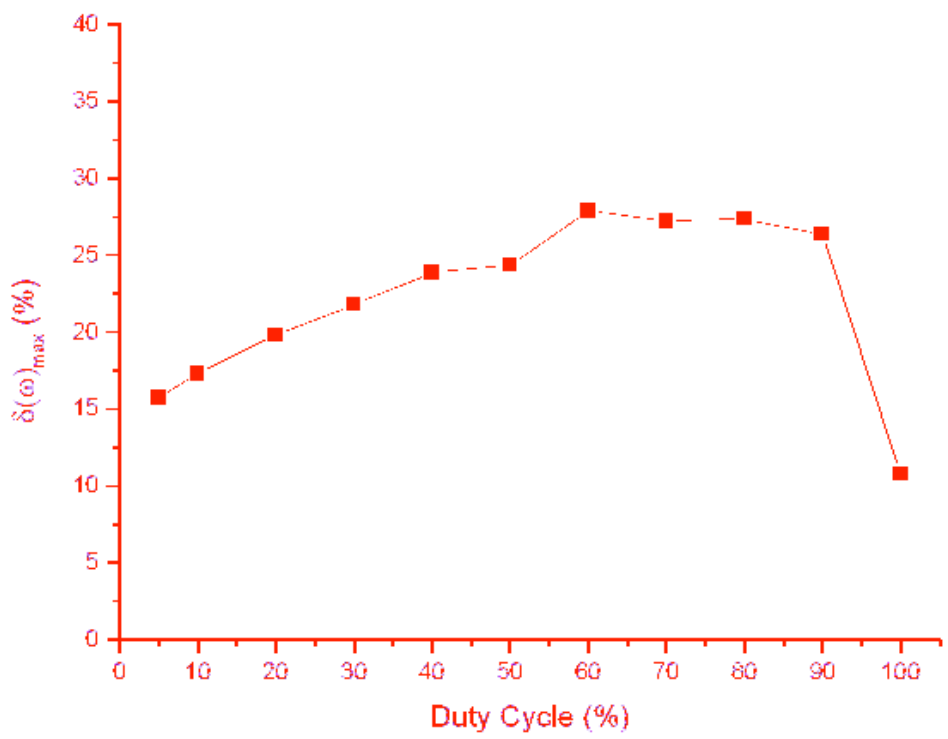

Fig. 26. Maximum relative reductions of total pressure loss coefficient with unsteady actuation of different duty cycles $\left(\nu_{\infty}=50 \mathrm{~m} / \mathrm{s}, i=0 \mathrm{deg}, V_{\mathrm{p}-\mathrm{p}}=10 \mathrm{kV}, F=23 \mathrm{kHz}, 70 \% \mathrm{Span}\right)$

It is found that there's also a duty cycle threshold in controlling the corner separation. When the duty cycle is less than $60 \%$, the control effect increases along with the duty cycle increasing. $\delta(\omega)_{\max }$ is $28 \%$ at the duty cycle of $60 \%$. Even when the duty cycle is $5 \%, \delta(\omega)_{\max }$ is $15.7 \%$, much more effective than the steady actuation. When the duty cycle is higher than $60 \%$, the control effect saturates along with the duty cycle increasing. Thus it can be inferred that when the duty cycle is less than $60 \%$, the injected energy is not sufficient to control the corner separation. In the separation control above a NACA 0015 airfoil with unsteady plasma aerodynamic actuation, the most effective duty cycle values range from $10 \%$ to $60 \%$. In the separation control of low-pressure turbine blades with unsteady plasma aerodynamic actuation, the lowest plasma duty cycle $(10 \%)$ was as effective as the highest plasma duty cycle $(50 \%)$ at the same excitation frequency. Thus, the optimum duty cycle also depends much on the flow separation state.

\subsection{Low speed axial compressor stability extension}

This series of tests were carried out using a low speed axial compressor test rig at Institute of Engineering Thermophysics, Chinese Academy of Sciences. The tested compressor rotor was isolated from the stator to avoid interaction effects generated by the presence of a downstream stator blade row. The isolated compressor rotor selected for this investigation is 
actually the rotor of the first stage of a low-speed three-stage axial compressor test rig, which has been used for a number of research programs for the flow instability in compression system. The blading is typical of high-pressure ratio compressor design. Previous work indicates that the isolated rotor is prone to tip stall behavior, which is suitable for flow control methods in the end wall flow regions.

The overall compressor performance in terms of pressure rise coefficient $\Psi$ and mass flow coefficient $\Phi$ was measured with eight static pressure taps on casing around the annulus in both the inlet and the outlet of the compressor. The measurement uncertainties were: static pressure, $\pm 60 \mathrm{~N} / \mathrm{m}^{2}$. Errors in calculated $\Psi$ and $\Phi$ were estimated at $\pm 0.2 \%$ maximum, as far as relative comparison between the results for a certain condition is concerned.

The basic principle of using plasma actuation reated caseing(PATC) to improve compressor stability range is shown in Fig. 27. When the PATC is energized, plasma forms and induces airflow along the direction of compressor inflow in the end wall flow region.

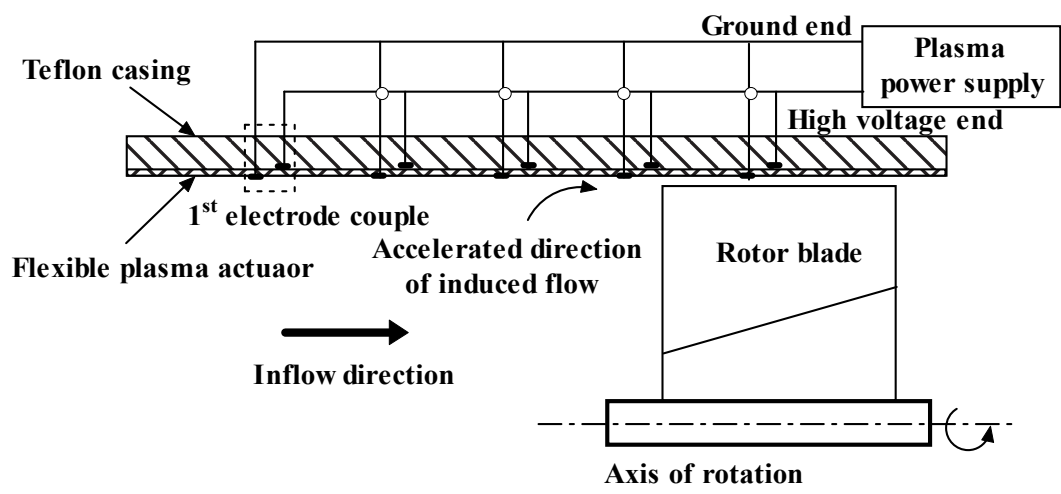

Fig. 27. Sketch map of using PATC to improve compressor stability range

The basic mechanism for plasma actuation to extend compressor's stability can be classified to three effects. The first is that plasma actuation induces air acceleration along with the inflow direction in the blade tip end wall region. Energy is added to the low-energy flow in the end wall region, which can increase mass flux at blade leading edge, inhibit development of blade tip secondary flow and leakage flow, and enhance circulating ability in the end wall region. Thus the accumulation of flow build up is minished. The second is that due to the end wall flow acceleration induced by plasma actuation, velocity in flow direction at blade tip channel is enhanced and inflow attack angle is reduced. Thus flow separation at blade suction surface is inhibited. The last effect is that plasma actuation is non-stationary and non-linear actuation, which can enhance mixture among flow with different momentum in the end wall region. Thus flow separation due to low energy is inhibited and compressor stability is extended. Since plasma actuation can minish flow build up extent in the blade tip end wall region, inhibit secondary flow and leakage flow, and enhance circulating ability, compressor pressure rise ability is improved.

PATC consists of a flexible plasma actuator and a casing. The plasma actuator, layout of which is asymmetrical, consists of 5 electrode couples. The $4^{\text {th }}$ electrode couple is located at $3 \mathrm{~mm}$ away from the blade leading edge, while the $5^{\text {th }}$ couple is located at the $40 \%$ blade tip chord. The thickness of teflon layer, $h$, is $0.5 \mathrm{~mm}$. The electrode is $0.035 \times 2 \mathrm{~mm}$ copper layer. 
The horizontal displacement between upper and lower electrode for each couple, $\Delta \mathrm{d}$, is 1 $\mathrm{mm}$. The distance between adjacent electrode couples, D, is $10 \mathrm{~mm}$. The casing is also made of teflon. Fig. 28 and Fig. 29 show the PATC and the low speed axial compressor with PATC, respectively. For the PATC and tested rotor, tip clearance is $0.6 \mathrm{~mm}$, which is $1.65 \%$ of the blade chord length.

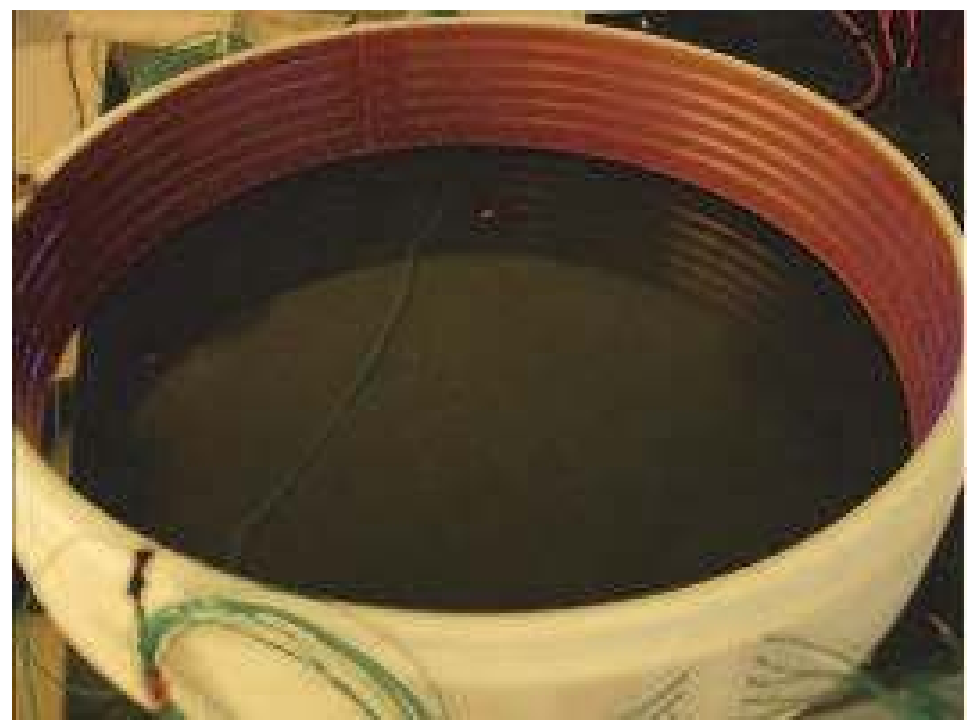

Fig. 28. Plasma actuation treated casing

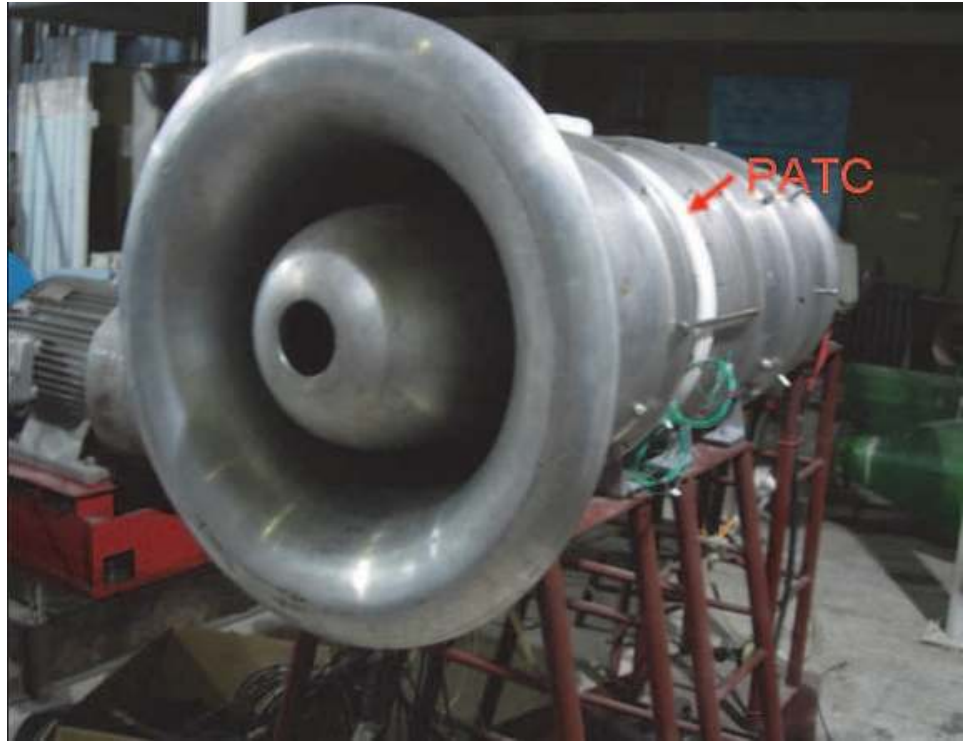

Fig. 29. Low speed axial compressor test rig with PATC 
Plasma actuation casing is energized by a high voltage power supply. The output of the power supply is sine wave. The amplitude and frequency range is $0-30 \mathrm{kV}$ and $6-40 \mathrm{kHz}$, respectively, which can be adjusted continuously.

The compressor throttling was throttled by the exit rotary cone valve mounted on the shaft and regulated manually when stall was approached. Wall static pressure was collected to calculate pressure rise coefficient $\Psi$ and flow coefficient $\Phi$, which are adopted as representatives of compressor performance and stability with and without plasma actuation at a constant rotor speed.

The effect of plasma actuation on the compressor performance and stability range is displayed in Fig. 30 at the rotor speed of $900 \mathrm{rpm}$. The $4^{\text {th }}$ electrode couple is actuated and the actuation voltage is $9 \mathrm{kV}$.

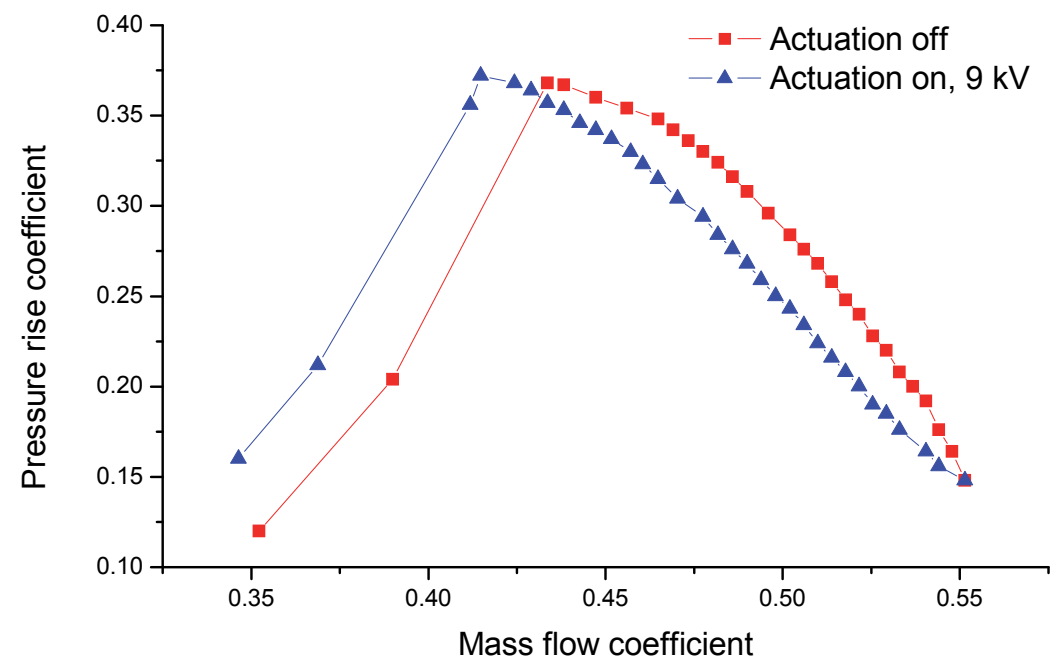

Fig. 30. Test results with and without plasma actuation(rotor speed: $900 \mathrm{rpm}$ )

The changes of maximum pressure rise coefficient, $\Psi_{\max }$ and mass flow coefficient near stall, $\Phi_{\mathrm{ns}}$ are summarized in table 1 . The $\Phi_{\mathrm{ns}}$ decreases by $5.2 \%$, while the $\Psi_{\max }$ increases by $1.08 \%$.

\begin{tabular}{|c|c|c|c|c|}
\hline & $\Psi_{\max }$ & $\Delta \Psi_{\max } / \Psi_{\max }$ & $\Phi_{\mathrm{ns}}$ & $\Delta \Phi_{\mathrm{ns}} / \Phi_{\mathrm{ns}}$ \\
\hline $\mathbf{0}$ & 0.3721 & & 0.4426 & \\
\hline $\mathbf{1}$ & 0.3761 & $1.08 \%$ & 0.4196 & $-5.2 \%$ \\
\hline
\end{tabular}

0: PATC off, 1: $4^{\text {th }}$ electrode couple on, $9 \mathrm{kV}$.

Table 1. The effect of plasma actuation on compressor performance and stability range

Fig. 31 illustrates the test results with and without plasma actuation at the rotor speed of $1080 \mathrm{rpm}$. When the $2^{\text {nd }}$ and 3 rd electrode couples are switched on, $\Phi_{\text {ns }}$ decreases by $1.42 \%$ and $5.07 \%$ when the actuation voltage is $9 \mathrm{kV}$ and $12 \mathrm{kV}$ respectively. $\Psi_{\max }$ decreases by $2.21 \%$ and $0.74 \%$ respectively. 


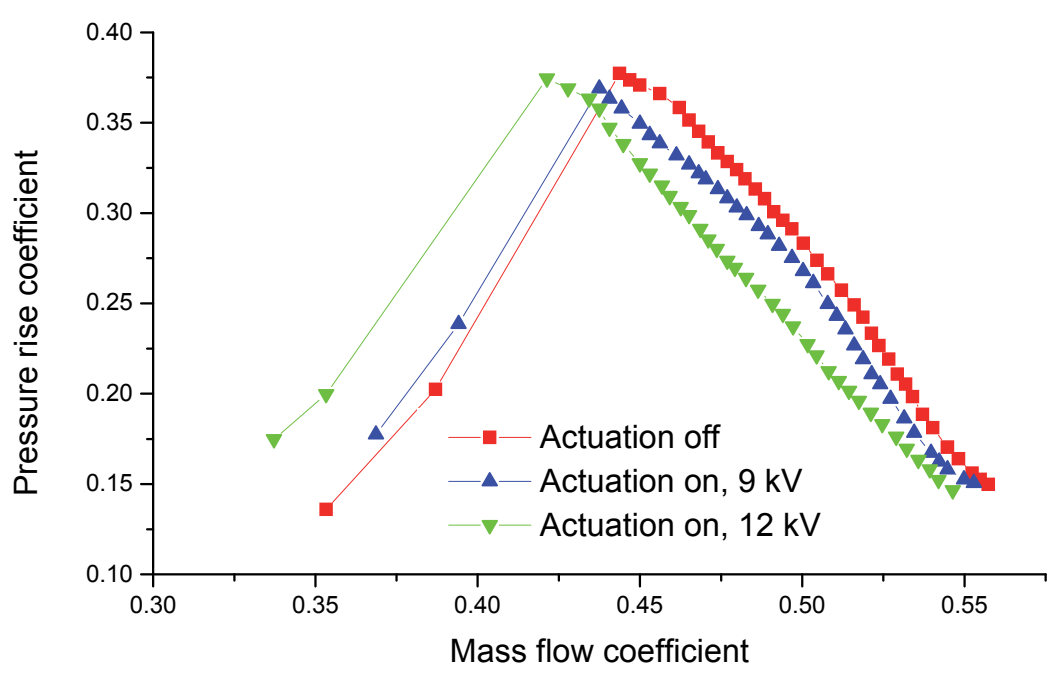

Fig. 31. Test results with and without plasma actuation(rotor speed: $1080 \mathrm{rpm}$ )

Along with the actuation voltage ascending, plasma actuation strength increases while plasma actuator's layout form and material remain same. Thus the velocity of induced flow acceleration increases, which can better enhance the circulation ability in end wall region, reduce inflow attack angle and promote flow mixture. As a result, the compressor stability range is much wider. Therefore plasma actuation strength, which can be improved by adjusting actuator layout form or increasing actuation voltage, is one key factor in plasma based stability extension.

Fig. 32 represents the effect of plasma actuation location on the compressor performance and stability range when the rotor speed equals $1080 \mathrm{rpm}$. When $3^{\text {rd }}$ and $4^{\text {th }}$ electrode couples are switched on at $12 \mathrm{kV}, \Phi_{\text {ns }}$ decreases by $1.42 \%$ and $\Psi_{\max }$ decreases by $1.47 \%$. When the $2^{\text {nd }}$ and $3^{\text {rd }}$ electrode couples are switched on, $\Phi_{\text {ns }}$ and $\Psi_{\max }$ decrease by $5.07 \%$ and $0.74 \%$, respectively. Therefore, different actuation location results in different stability range extension effect. One possible reason is that the $4^{\text {th }}$ electrode couple is just $3 \mathrm{~mm}(8.3 \%$ of axial chord) away from the rotor blade leading edge, where flow build up is very serious and flow separation has well developed in blade tip end wall region at near stall state. Thus plasma actuation at this location can't control the flow field well and the stability extension effect is limited. When the $2^{\text {nd }}$ and $3^{\text {rd }}$ electrode couple is on, because the $3^{\text {rd }}$ electrode couple is $18 \mathrm{~mm}(49.5 \%$ of axial chord) away from the rotor blade leading edge, plasma actuation can accelerate the flow boundary layer before flow separation and build up in well development, which can inhibit the end all separation flow, secondary flow and leakage vortex better. Therefore stability extension effect is much better.

The changes of $\Psi_{\max }$ and $\Phi_{\mathrm{ns}}$ are summarized in table 2. $\Psi_{\max }$ decreases at every case when plasma actuation is on. The $\Psi_{\max }$ decrease is least when the $\Phi_{\mathrm{ns}}$ decrease is most. So there is no contradiction between stability range extension and pressure rise coefficient improvement. When the ability for plasma actuation to control the blade tip end wall region flow becomes stronger, the stability extension effect is better and the pressure rise ability almost remains same. 


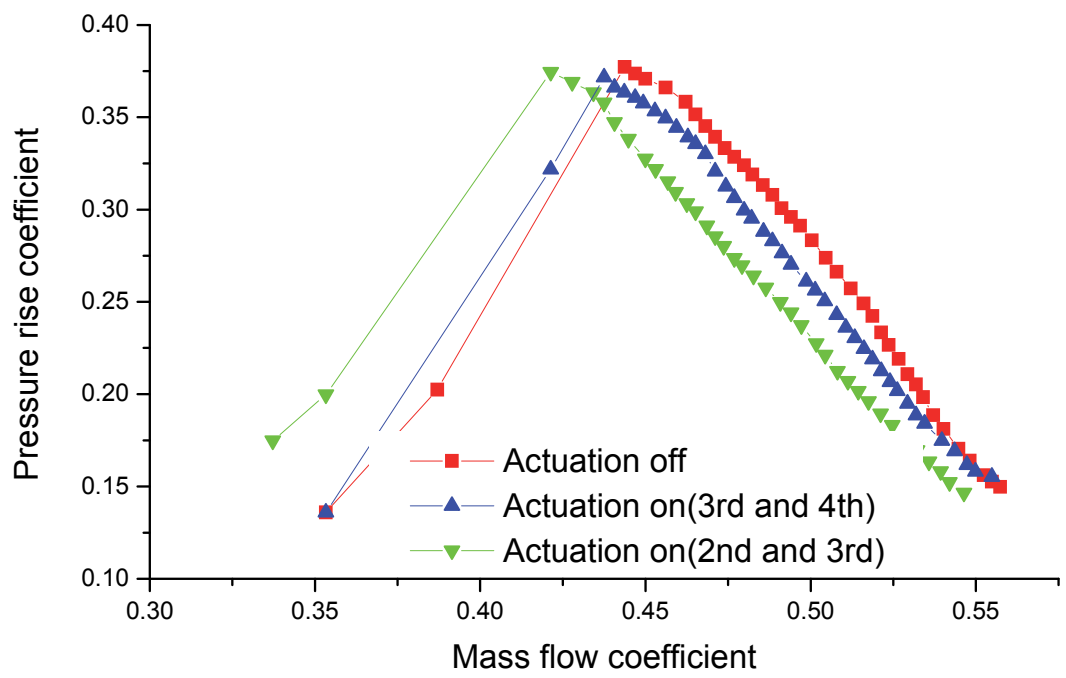

Fig. 32. Test results with and without plasma actuation (rotor speed: $1080 \mathrm{rpm}$ )

\begin{tabular}{|c|c|c|c|c|}
\hline & $\Psi_{\max }$ & $\Delta \Psi_{\max } / \Psi_{\max }$ & $\Phi_{\mathrm{ns}}$ & $\Delta \Phi_{\mathrm{ns}} / \Phi_{\mathrm{ns}}$ \\
\hline $\mathbf{0}$ & 0.3772 & & 0.4438 & \\
\hline $\mathbf{1}$ & 0.3689 & $-2.21 \%$ & 0.4375 & $-1.42 \%$ \\
\hline $\mathbf{2}$ & 0.3744 & $-0.74 \%$ & 0.4213 & $-5.07 \%$ \\
\hline $\mathbf{3}$ & 0.3717 & $-1.47 \%$ & 0.4375 & $-1.42 \%$ \\
\hline
\end{tabular}

0 : PATC off.

1: $2^{\text {nd }}$ and $3^{\text {rd }}$ electrode couples on, $9 \mathrm{kV}$.

2: $2^{\text {nd }}$ and $3^{\text {rd }}$ electrode couples on, $12 \mathrm{kV}$.

$3: 3^{\text {rd }}$ and $4^{\text {th }}$ electrode couples on, $12 \mathrm{kV}$.

Table 2. The effect of plasma actuation on compressor performance and stability range

\section{Supersonic plasma flow control}

Based on plasma aerodynamic actuation, plasma flow control is a novel active flow control technique and has important applications in the field of supersonic flow control. Shock waves are typical aerodynamic phenomena in supersonic flow. If they are controlled effectively, the aerodynamic performance of both flight vehicles and aeroengines will be greatly enhanced. Conventional mechanical or gasdynamic control methods have disadvantages of complex structure and slow response. Novel plasma flow control method has advantages of simple structure, fast response and wide actuation frequency range. Therefore, plasma flow control method has become a newly-rising focus in the field of shock wave control.

\subsection{Experimental principle and arrangement}

Fig. 33 shows the MHD flow control experimental principle. The high density plasma column which primarily consists of ions and electrons was generated between a pair of electrodes 
through pulsed DC discharge. There were three pairs of electrodes and an oblique shock wave appeared in front of the ramp in low-temperature supersonic flow. The alphabet " $\mathrm{I}$ " and " $\mathrm{B}$ " represented the current and magnetic field. The arrows gave their directions.

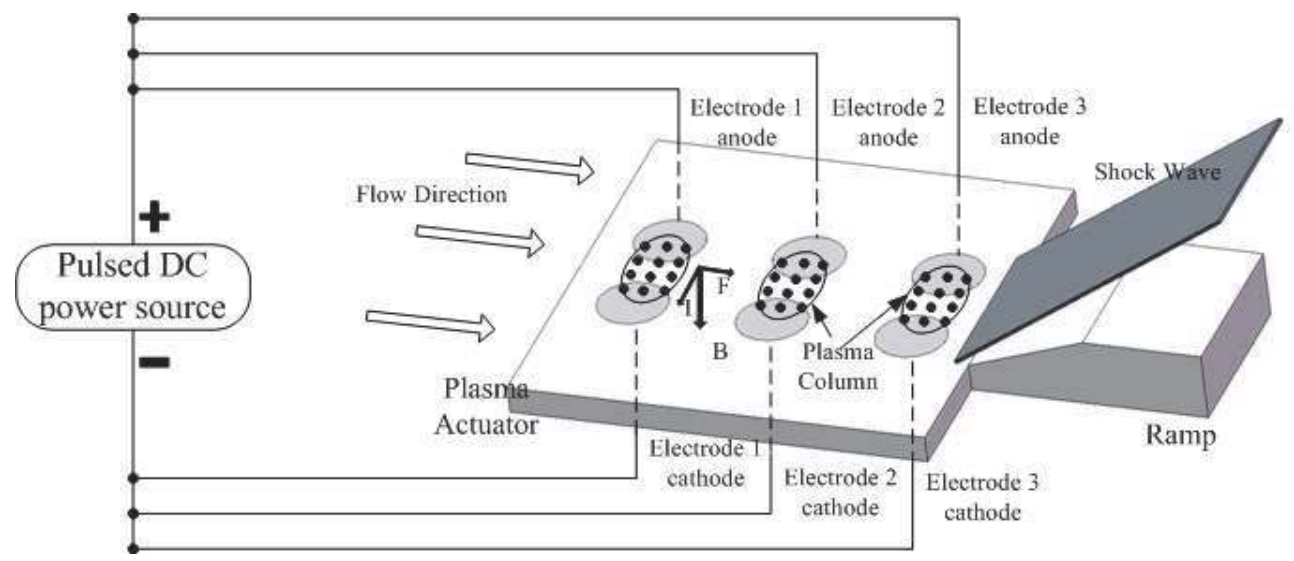

Fig. 33. The experimental principle

When magnetic field, normal to the surface, was imposed on the plasma column created in the boundary layer, it affected both the plasma and, through the Lorentz body force $(j \times B$ body force), the flow. The direction of Lorentz body force was determined by the directions of current and magnetic field. The alphabet " $F$ " represented the Lorentz body force which could accelerate the flow.

The plasma column was produced by pulsed DC discharge. Therefore the plasma would be influenced by electric field force, magnetic field force and the airflow inertial force. The magnetic field force and the airflow inertial force were dominant. When the direction of magnetic field force was same as that of airflow inertial force and the velocity of plasma was faster than that of the neutral gas molecules, the plasma would strike the neutral gas molecules to transfer momentum and accelerate the flow in the boundary layer. Otherwise, when the direction of magnetic field force was against with that of airflow inertial force, the plasma would strike the neutral gas molecules to transfer momentum and decelerate the flow in the boundary layer.

MHD flow control system consisted of low-temperature supersonic wind tunnel, plasma actuation system, experimental ramp, magnetic field generator, parameter measurement system and schlieren optical system. The inlet total pressure of low-temperature supersonic wind tunnel was about 5-7atm. The stagnation conditions for the tunnel were atmospheric pressure and room temperature. The run time could reach up to 60 seconds dependent on the inlet total pressure. The experimental duct was $115 \mathrm{~mm}$ (length) $\times 80 \mathrm{~mm}$ (width) and the designed Mach number was 2.2. The static pressure was 0.5-0.7atm and the static temperature was $152 \mathrm{~K}$.

The plasma actuation system included pulsed DC power source, plasma actuator, insulating acrylic base. Pulsed DC power source was the critical equipment which consisted of high voltage pulsed circuit, high voltage DC circuit and feedback circuit. It could provide $0-90 \mathrm{kV}$ selected high voltage pulse and $0-3 \mathrm{kV}$ selected high voltage direct current. The electrodes were made of plumbago, and were flush-mounted on the top wall of the insulating 
dielectric. The diameter of the electrode was $10 \mathrm{~mm}$. The insulating dielectric was made of $\mathrm{BN}$ ceramic. Two kinds of arrangements for the plasma actuator were adopted according to the distance between a pair of electrodes $(D=5 \mathrm{~mm}$ or $D=8 \mathrm{~mm})$.

The experimental ramp was also constructed of insulating acrylic material. It was installed on the insulating acrylic base. The dimension of the ramp was $34 \mathrm{~mm}$ (length) $\times 25 \mathrm{~mm}$ (width) $\times 6 \mathrm{~mm}$ (height). One angle of ramp was $A=15^{\circ}$ and another was $A=20^{\circ}$. As illustrated in Fig. 34, 10 static pressure measurement holes were drilled on the acrylic base, the plasma actuator and the ramp. The holes were numbered with k1-k10 from upstream to downstream. Holes k2-k8 were drilled on the plasma actuator, and holes $\mathrm{k} 9-\mathrm{k} 10$ were drilled on the ramp. The diameter of $\mathrm{k} 1-\mathrm{k} 10$ was $0.5 \mathrm{~mm}$. On the plasma actuator the distance between adjacent holes was $10 \mathrm{~mm}$ except that the distance between $\mathrm{k} 2$ and $\mathrm{k} 3$ was $7.5 \mathrm{~mm}$ and the distance between $\mathrm{k} 7$ and $\mathrm{k} 8$ was also $7.5 \mathrm{~mm}$. On the ramp the distance between $\mathrm{k} 9$ and plasma actuator edge was $6 \mathrm{~mm}$ and the distance between $\mathrm{k} 9$ and $\mathrm{k} 10$ was $6 \mathrm{~mm}$.

$\mathrm{Nd}-\mathrm{Fe}-\mathrm{B}$ rare-earth permanent magnets were used as the magnetic field generator which was located normal to experimental duct. The direction of magnetic field was perpendicular to the flow direction and the electric field direction. The magnetic field strength was about $0.3 \mathrm{~T}$ between two magnets.

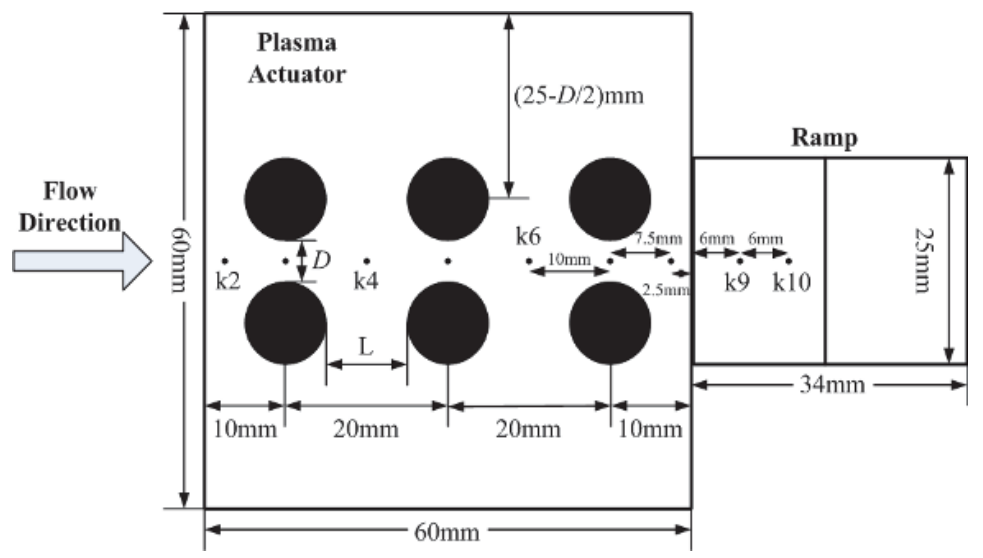

Fig. 34. The dimensions of the plasma actuator and the ramp

Parameter measurement system consisted of electric parameter and flow characteristic measurement systems. Electric parameter measurement system included oscillograph (DPO4104, Tektronix Inc.), high voltage probe (P6015A, Tektronix Inc.) and current probe(TCP312+TCPA300, Tektronix Inc.). Flow characteristic measurement system included 10 static pressure sensors and a data collection apparatus. Because the run time of the wind tunnel was above 10 seconds in every experiment, the inlet total pressure decreased slowly during the experimental time. Therefore, in this study the ratio of Pitot pressure after shockwave to that before shock-wave was adopted to compare the flow characteristic of the airflow around the ramp that was illustrated as $P_{k 10} / P_{k 7}$.

Schlieren optical system consisted of a high-speed camera and a storage computer. The highspeed camera was an Optronis ${ }^{\circledR}$ high-speed camera, and the maximum frame frequency was 200k frames per second(FPS). In this study, the schlieren pictures were taken at 8kFPS. The exposure time was $100 \mu$ s and the run time was $8 \mathrm{~s}$. 


\subsection{Experimental results}

In terms of different magnetic field directions, distances between electrodes, ramp angles and DC Voltage, the results of these four kinds of MHD flow control were compared and analyzed.

Through changing the magnetic field direction, MHD acceleration and MHD deceleration experiments were carried out. Fig. 35 represented two typical flow characteristics.

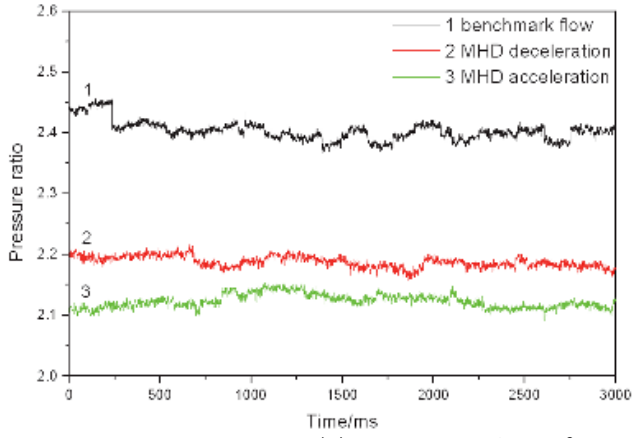

(a) $\mathrm{D}=5 \mathrm{~mm}, \mathrm{~A}=20^{\circ}$

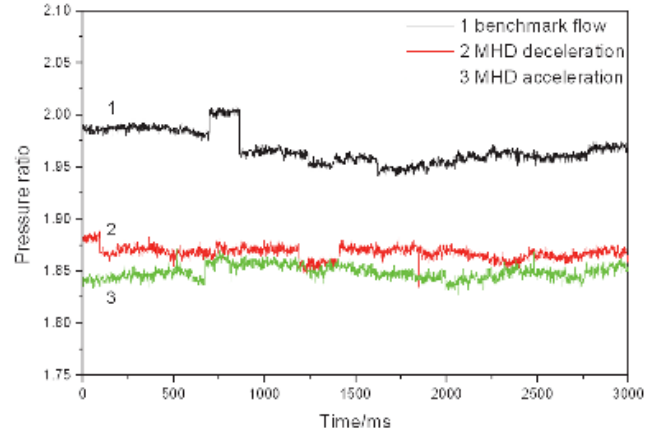

(b) $\mathrm{D}=8 \mathrm{~mm}, \mathrm{~A}=15^{\circ}$

Fig. 35. Two typical flow characteristics

The time-averaged pressure ratio decreased by $6.04 \%$ and $5.09 \%$ with MHD acceleration and MHD deceleration respectively. Thus, MHD flow control could drastically weaken the oblique shock wave strength and change the flow characteristic of the airflow around the ramp. MHD acceleration was more effective than MHD deceleration.

MHD acceleration experiments were carried out at ramp angle $A=20^{\circ}$ with $D=8 \mathrm{~mm}$ or $5 \mathrm{~mm}$. Fig.36 showed the flow characteristics with different distances. At $D=8 \mathrm{~mm}$ the timeaveraged pressure ratio decreased by $19.66 \%$ with MHD acceleration. At $D=5 \mathrm{~mm}$ the timeaveraged pressure ratio decreased by $11.64 \%$ with MHD acceleration. Thus, MHD acceleration was more effective to weaken the shock wave strength when $D$ increased, but there existed a maximum value $D_{\max }$ exceeding which the airflow could not be ionized restricted by power source.

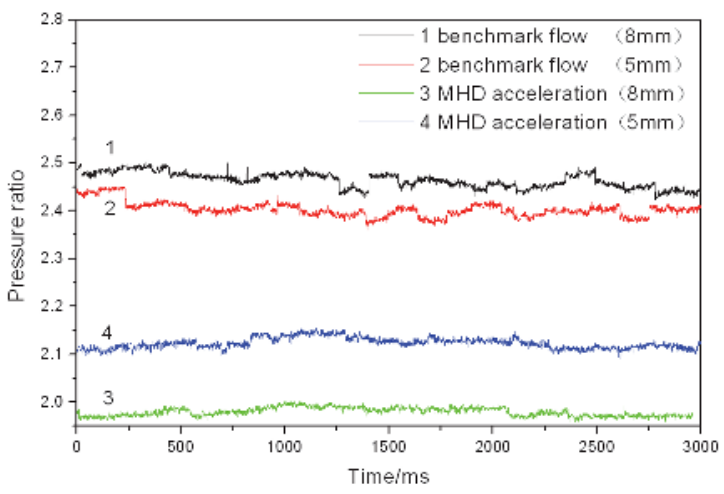

Fig. 36. Flow characteristics with different distances 
MHD acceleration experiments were carried out at $D=8 \mathrm{~mm}$ with different ramp angles $A=15^{\circ}$ or $20^{\circ}$. Fig. 37 showed the flow characteristics with different ramp angles. At $A=15^{\circ}$ the time-averaged pressure ratio decreased by $6.04 \%$ with MHD acceleration. At $A=20^{\circ}$ the time-averaged pressure ratio decreased by $19.66 \%$ with MHD acceleration. Thus, MHD acceleration was more effective to weaken the shock wave strength when $A$ increased, but there existed a maximum $A_{\max }$ exceeding which oblique shock wave strength would be too strong to be changed.

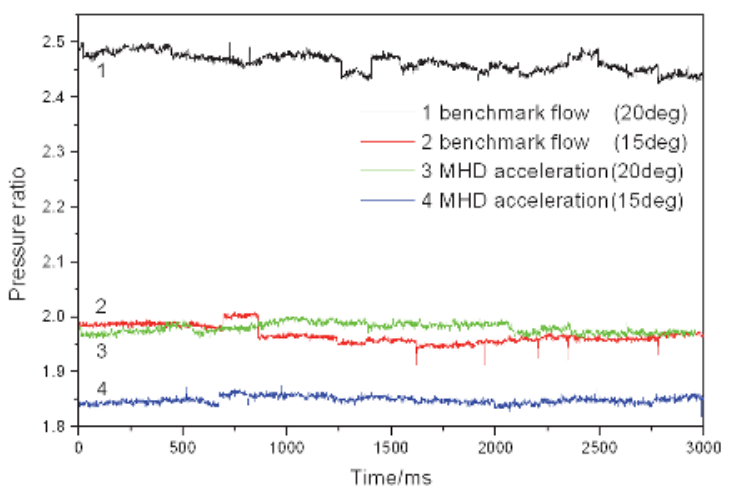

Fig. 37. Flow characteristics with different ramp angles

Through changing DC voltage $\mathrm{V}_{\mathrm{DC}}(2 \mathrm{kV}, 2.5 \mathrm{kV}, 3 \mathrm{kV})$, MHD acceleration and MHD deceleration experiments were carried out at ramp angle $D=8 \mathrm{~mm}, A=15^{\circ}$. Fig. 38 showed the flow characteristics with different $V_{D C}$. Fig. 38(a) showed the static pressure ratio varying with MHD acceleration. The time-averaged pressure ratio decreased by $3.95 \%$, $5.19 \%$ and $6.04 \%$ with $\mathrm{V}_{\mathrm{DC}}=2 \mathrm{kV}, 2.5 \mathrm{kV}$ and $3 \mathrm{kV}$ respectively. Fig. $38(\mathrm{~b})$ showed the static pressure ratio varying with MHD deceleration. The time-averaged pressure ratio decreased by $3.44 \%, 4.26 \%$ and $5.09 \%$ with $\mathrm{V}_{\mathrm{DC}}=2 \mathrm{kV}, 2.5 \mathrm{kV}$ and $3 \mathrm{kV}$ respectively. Thus, MHD flow control could drastically weaken the oblique shock wave strength and change the flow characteristic of the airflow around the ramp. MHD interaction was more effective when $V_{D C}$ increased.

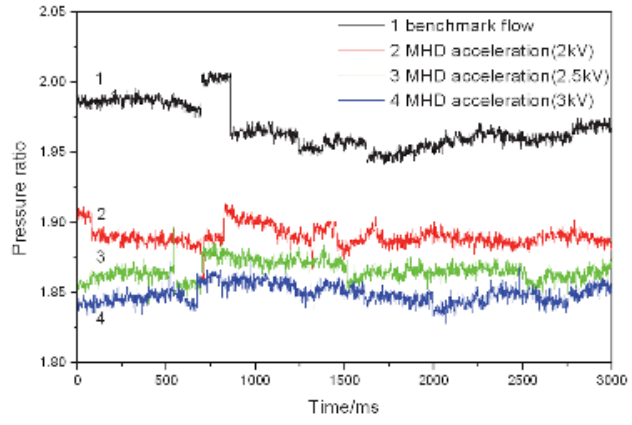

(a) MHD acceleration

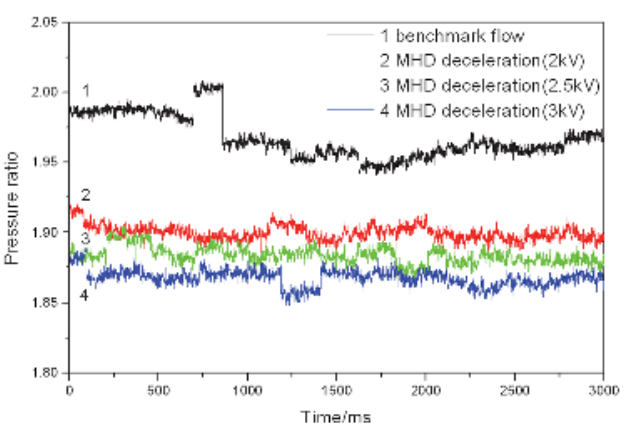

(b) MHD deceleration

Fig. 38. Flow characteristics with different $V_{D C}$ 
The schlieren pictures were captured during the time of flow characteristic measurement at all conditions. The typical schieren pictures were illustrated in Fig. 39 at $D=8 \mathrm{~mm}, A=20^{\circ}$ with MHD acceleration. Fig. 39(a) showed the benchmark flow with no electric field and no magnetic field while Fig. 39(b) showed the flow with electric field and magnetic field in the case of MHD acceleration. Four shock waves before the ramp were shown in the benchmark picture, three of which were produced by the coarse interface between electrodes and ceramic and the last shock wave was produced by the ramp. Without MHD acceleration the shock wave angle near the ramp was about $37.5^{\circ}$, and the distance between the shock wave location and the ramp was about $7.1 \mathrm{~mm}$. With MHD acceleration the shock wave angle near the ramp was reduced to $35^{\circ}$, and the distance was increased to $10 \mathrm{~mm}$ which meant the shock wave was moved upstream by $2.9 \mathrm{~mm}$. Therefore, MHD flow control could change shock wave location, convert a strong shock wave into many weak shock waves, weaken the shock wave strength and change the flow characteristic near the ramp.

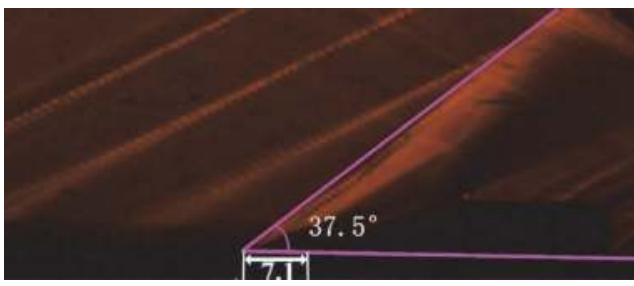

(a) Benchmark flow

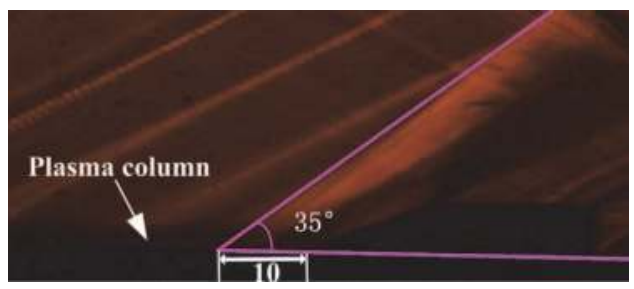

(b) MHD acceleration flow

Fig. 39. Benchmark and MHD acceleration flow at a typical condition

\section{Conclusion}

The principle of plasma aerodynamic actuation and its application in subsonic and supersonic flow control was summarized. The mechanism for plasma flow control can be summarized as momentum effect, shock effect, and chemical effect. Both the plasma and flow characteristics of the plasma aerodynamic actuation were investigated. Plasma flow control used in airfoil separation control, corner separation control, axial compressor stability extension and shock wave control were studied.

\section{Acknowledgment}

The authors thank Yikang Pu, Shouguo Wang, Junqiang Zhu and Chaoqun Nie for the help in the experiment and analysis. Support for this work was received from the National Natural Science Foundation of China (50906100, 10972236, 51007095) is gratefully acknowledged.

\section{References}

Corke, T., Post, M. \& Orlov, D. Dielectric Barrier Discharge Plasma Actuators for Flow Control, Progress in Aerospace Sciences, Vol.43, (January 2010), pp. 505-529, ISSN 0376-0421 
Moreau, E. Airflow Control by Non-thermal Plasma Actuators, Journal of Physics D: Applied Physics, Vol.40, No.3, (January 2007), pp. 605-636, ISSN 0022-3727

Roth, J. Aerodynamic Flow Acceleration Using Paraelectric and Peristaltic Electrohydrodynamic Effects of a One Atmosphere Uniform Glow Discharge Plasma, Physics of Plasmas, Vol.10, No.5, (May 2003), pp. 2117-2126, ISSN 1070$664 X$

Bletzinger, P., Ganguly, B. \& VanWie, D. (2005). Plasmas in High Speed Aerodynamics, Journal of Physics D: Applied Physics, Vol.38, No.4, (April 2005), pp. R33-57, ISSN 0022-3727

Wu, Y., Li, Y., Jia, M., Song, H., Guo, Z. G., Zhu, X. \& Pu, Y. Influence of Operating Pressure on Surface Dielectric Barrier Discharge Plasma Aerodynamic Actuation Characteristics, Applied Physics Letters, Vol.93, No.3, (July 2008), pp. 031503(3p), ISSN 0003-6951

Li, Y., Wu, Y., Zhou, M., Su, C., Zhang, X. \& Zhu, J. Control of the Corner Separation in a Compressor Cascade by Steady and Unsteady Plasma Aerodynamic Actuation, Experiments in Fluids, Vol.48, No.6, (June 2010), pp. 1015-1023, ISSN 0723-4864

Li, Y., Wu, Y., Jia, M., Zhou, Z., Guo, Z., \& Pu, Y. Optical Emission Spectroscopy Investigation of a Surface Dielectric Barrier Discharge Plasma Aerodynamic Actuator, Chinese Physics Letters, Vol.25, No.11, (November 2008), pp. 4068-4071, ISSN 0256-307X

Wu, Y., Li, Y., Jia, M., Song, H., Su, C. \& Pu, Y. Experimental Investigation into Characteristics of Plasma Aerodynamic Actuation Generated by Dielectric Barrier Discharge, Chinese Journal of Aeronautics, Vol.23, No.1, (January 2010), pp. 39-45, ISSN 1000-9361

Li, Y., Wang, J., Wang, C., An, Z., Hou, S. \& Xing, F. Properties of Surface Arc Discharge in a Supersonic Airflow, Plasma Sources Science and Technology, Vol.19, No.2, (March 2010), pp.025016(9p), ISSN 0963-0252

Wang, J., Li, Y. \& Xing, F. Investigation on Oblique Shock Wave Control by Arc Discharge Plasma in Supersonic Airflow, Journal of Applied Physics, Vol.106, No.7, (October 2009), pp.073307(7p) , ISSN 0021-8979

Wang, J., Li, Y., Cheng, B., Su, C., Song, H. \& Wu, Y. Effects of Plasma Aerodynamic Actuation on Oblique Shock Wave in a Cold Supersonic Flow, Journal of Physics D: Applied Physics, Vol.42, No.16, (June 2009), pp. 165503(8p), ISSN 0022-3727

$\mathrm{Su}, \mathrm{C} ., \mathrm{Li}, \mathrm{Y} ., \mathrm{Cheng}$, B., Wang, J., Cao, J. \& Li, Y. Experimental Investigation of MHD Flow Control for the Oblique Shock Wave Around the Ramp in Low-temperature Supersonic Flow, Chinese Journal of Aeronautics, Vol.23, No.1, (January 2010), pp. $22-$ 32, ISSN 1000-9361

Wu, Y., Li, Y., Zhu, J., Su, C., Liang, H. \& Li, G. (2007). Experimental Investigation of a Subsonic Compressor with Plasma Actuation Treated Casing, 37th AIAA Fluid Dynamics Conference and Exhibit, pp. 1-8, Miami, FL, USA, June 25-28, 2007

Li, Y., Wu, Y., Liang, H., Song, H. \& Jia, M. (2010), The mechanism of plasma shock flow control for enhancing flow separation control capability, Chinese Sci Bull (Chinese Ver), vol. 55, No. 31, (November 2010), pp. 3060- 3068, ISSN 0023-074X 
Li, Y., Liang, H., Ma, Q., Wu, Y., Song, H., \& Wu, W.(2008). Experimental investigation on airfoil suction side flow separation suppression by pulse plasma aerodynamic actuation, Acta Aeronautica et Astronautica Sinica (Chinese Ver), vol. 29, No. 6, (November 2008), pp. 1429-1435, ISSN 1000-6893 


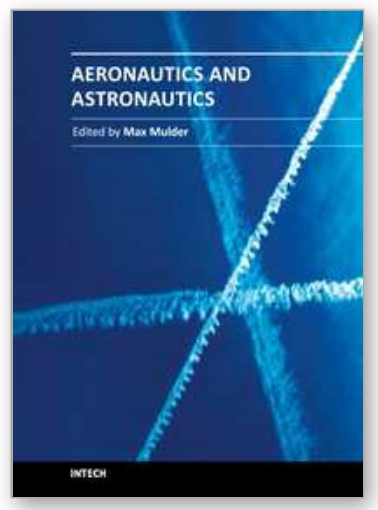

\author{
Aeronautics and Astronautics \\ Edited by Prof. Max Mulder
}

ISBN 978-953-307-473-3

Hard cover, 610 pages

Publisher InTech

Published online 12, September, 2011

Published in print edition September, 2011

In its first centennial, aerospace has matured from a pioneering activity to an indispensable enabler of our daily life activities. In the next twenty to thirty years, aerospace will face a tremendous challenge - the development of flying objects that do not depend on fossil fuels. The twenty-three chapters in this book capture some of the new technologies and methods that are currently being developed to enable sustainable air transport and space flight. It clearly illustrates the multi-disciplinary character of aerospace engineering, and the fact that the challenges of air transportation and space missions continue to call for the most innovative solutions and daring concepts.

\title{
How to reference
}

In order to correctly reference this scholarly work, feel free to copy and paste the following:

Ying-hong Li, Yun Wu, Hui-min Song, Hua Liang and Min Jia (2011). Plasma Flow Control, Aeronautics and Astronautics, Prof. Max Mulder (Ed.), ISBN: 978-953-307-473-3, InTech, Available from: http://www.intechopen.com/books/aeronautics-and-astronautics/plasma-flow-control

\section{INTECH}

open science | open minds

\section{InTech Europe}

University Campus STeP Ri

Slavka Krautzeka 83/A

51000 Rijeka, Croatia

Phone: +385 (51) 770447

Fax: +385 (51) 686166

www.intechopen.com

\section{InTech China}

Unit 405, Office Block, Hotel Equatorial Shanghai

No.65, Yan An Road (West), Shanghai, 200040, China

中国上海市延安西路 65 号上海国际贵都大饭店办公楼 405 单元

Phone: +86-21-62489820

Fax: +86-21-62489821 
(C) 2011 The Author(s). Licensee IntechOpen. This chapter is distributed under the terms of the Creative Commons Attribution-NonCommercialShareAlike-3.0 License, which permits use, distribution and reproduction for non-commercial purposes, provided the original is properly cited and derivative works building on this content are distributed under the same license. 